\title{
May, Walther
}

Die von Stuhlmann im Jahre 1889 gesammelten ostafrikanischen Alcyonaceen des Hamburger Museums 



\title{
Die von \\ Dr. Stuhlmann im Jahre 1889 gesammelten ostafrikanischen Alcyonaceen des Hamburger Museums.
}

\section{Inaugural-Dissertation} zur Erlangung der Doktorwürde a der philosophischen Fakultät der Universität Jena.

\author{
Von \\ Walther May.
}

Ha mburg 1898. 



\section{Die von}

Dr. Stuhlmann im Jahre 1889 gesammelten

\section{ostafrikanischen Alcyonaceen}

des Hamburger Museums.

\section{Inaugural-Dissertation} zur Erlangung der Doktorwürde in der philosophischen Fakultät der Universität Jena.

Von

Walther May.

Ha mburg 1898. 


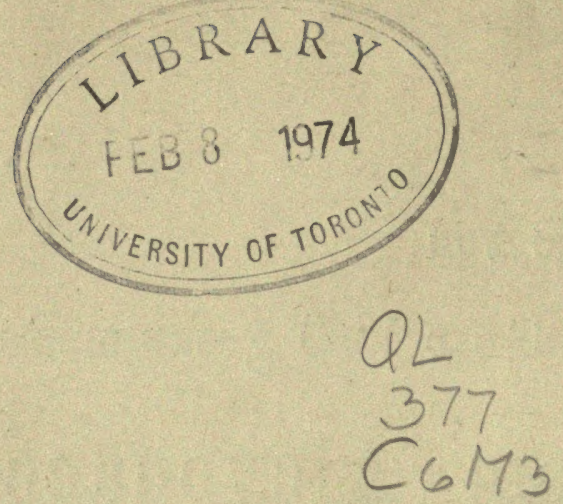

Genehmigt von der philosophischen Fakultät der Universität Jena, auf Antrag des Herrn Professors Dr. Haeckel.

Jena, den 8. Juli 1898.

Geh. Hofrat Prof. Dr. Thomae, d. Z. Dekan. 
Durch gütige Vermittlung von Herrn Professor KürenthaL in Jena ist mir von dem Naturhistorischen Museum in Hamburg das von Dr. STuHLMasa im Jahre 1889 gesammelte reiche Material an ostafrikanischen Alcyonaceen zur Bearbeitung überlassen worden. Gleichzeitig wurde mir von dem Berliner Museum für Naturkunde eine schöne Sammlung von Alcyonaceen aus verschiedenen Erdgegenden zur Verfügung gestellt. Dieses umfangreiche Material gab mir Veranlassung zu einer eingehendern Revision der ganzen Alcyonaceengruppe, die mit Tafeln versehen in der Jenaischen Zeitschrift für Naturwissenschaft erscheinen wird. Hier gebe ich einen kurzen Auszug aus jener gröfern Arbeit, der nur die von StuhLasans gesammelten ostafrikanischen Alcyonaceen des Hamburger Museums umfaßst.

Das StuhLManx'sche Material stammt größtenteils aus der Meeresstraße zwischen Kokotoni auf Sansibar, und der Insel Tumbatu und enthält 36 Arten: 7 Clavulariiden, 1 Telestide, 2 Tubiporiden, 9 Xeniiden, 5 Aleyoniiden und 12 Nephthyiden. Davon erwiesen sich als neu: 4 Clavulariiden, 5 Xeniiden, 1 Alcyoniide und 11 Nephthyiden, also im ganzen 21 Spezies. Besonders erwähnenswert erscheint mir die große Zahl neuer Arten der Nephthyidengattung Ammothea. Die Zahl der bisher bekannten Arten dieser Gattung betrug 7, in StuHLisans's Kollektion befanden sich nicht weniger als 11 neue. Es wird somit die von KüKENTHAL in seiner Bearbeitung der Nephthyiden von Ternate ausgesprochene Vermutung bewahrheitet, daßs weitere eingehendere systematische Untersuchungen der Nephthyidenfaunen indopacifischer Küstengebiete noch einen ungeahnten Reichtum an neuen Formen zu Tage fördern würden.

\section{Unterordnung: Alcyonacea Verrill.}

Synonyma: Lithophyta (pars) + Zoophyta fixata (pars), Lissḱ.

Zoophyta (pars), Pallas.

Cellulana calcarea (pars) + C. fungosa (pars), O. Fr. MÜLlekr.

Eponges (pars), Cuvier.

Polypi vaginati (pars) + Polypi tubiferi, Lasrarck.

Polypes à tuyaux (pars) + Polypes à corticaux (pars), Cuvier. 
Tubiporées (pars) + Alcyonées (pars), Lamouroux.

Tubiporoea + Alcyonaria, BlainviLle.

Zoocorallia Oetactinia (pars), Ehrenberg.

Alcyonidae + Cornularidae + Tubiporidae, Dana.

Alcyonidae, Johnston.

Alcyonidae, Milne-Edwards.

Sarcophyta (pars), Gray.

Alcyonides malacodermes, Duchassaing et Michelotti.

Alcyonidae + Briaraceae (pars), KöLLIKER.

Alcyonacea, VerriLL.

Zoophytaria carnosa, Gray.

Alcyonidae (excl. Haimeinae), Kuunzinger.

Alcyonacea (excl. Haimeidae), Studer.

Alcyonida, Danielssen.

Alcyonacea (excl. Haimeidae), Wright und Studer.

Alcyonacea (excl. Haimeidae), v. Косн.

Stolonifera + Alcyonacea, Hickson.

Alcyonacea (excl. Haimeidae), KÜKenthaL.

Sarcophytaria (excl. Monoxenida) + Tubiporaria, HAEckel.

Diagnose: Die Alcyonaceen sind festgewachsene soziale Octocorallen mit mesodermalem Stückel- oder Röhrenskelett. In einzelnen Fällen fehlt das Skelett ganz, oder es ist nur ein ektodermales Hornskelett vorhanden.

Systematische Charaktere: Die natürliche Systematik der Alcyonaceen berücksichtigt hauptsächlich folgende Verhältnisse: 1) das Verhalten der Stolonen, 2) den Habitus der Kolonie, 3) die Retraktionsfähigkeit der Polypen, 4) die Anordnung der Polypen, 5) die Arbeitsteilung der Polypen, 6) die Skelettbildung, 7) die Tentakelbildung, 8) die Form der Polypen, 9) die Größe der Polypen, 10) die Farbe der Kolonien und ihrer Teile. Von diesen Charakteren sind 1 und 2 wesentlich Familiencharaktere, 3,4 und 5 wesentlich Gattungscharaktere und die übrigen Artcharaktere.

\section{Familie: Clavulariidae Hickson.}

\section{Litteratur:}

1) LAMOUROUX, Bulletin de la Soc. philomatique 1812.

2) LAMOUROUX, Polyp. flexibles 1816. S. 232.

3) LAMARCK, Hist. nat. des animaux sans vertèbres. Tome II. Paris 1816. S. 111 und $40 \%$.

4) SAVIGNY, Description de l'Egypte. Hist, nat. Tome I, 4. partie. Paris. S. 228. Pl. T. II. Paris 1817, Polypes, Pl. I, Fig. 4-7.

5) LAMOURouX, Expos. méthodique des genres de l'ordre des Polypiers. Paris 1821. S. 17 .

6) LESSON, Voyage de la Coquille. Zooph. 1825. S. 87.

7) BLAINVILLE, Dict. d. Sei. Nat. T. X, 1830. S. 499. 
8) QUOY et GAIMARD, Voyage de découvertes de l'Astrolabe. ZooI. Paris 1833. S. $260 \mathrm{ff}$. Pl. 21, Fig. $10-12,13-16$.

9) EHRENBERG, Die Korallentiere des roten Meeres, Berlin 1834. S. 54, 55 und 61.

10) BLAINVILLE, Manuel d'Actinologie. Paris 1834. S. 498.

11) JOHNSTON, History of Britjsh Zoophytes. T. I. 1847. S. 179.

12) PHILIPPI, Zool. Beob. Archiv f. Naturg. 1842, T. I. S. 36.

13) DANA, Zoophytes. Philadelphia 1846. S. 627.

14) KOREN og DANIELSSEN, Fauna littoralis norvegicae. Part. II. 1856. S. 65.

15) MILNE-EDWARDS, Histoire nat, des Coralliaires. Tome I, Paris 1857. S. $104 \mathrm{ff}$.

16) DANA, Synopsis of the Report on Zoophytes etc. New Haven 1859. S. $120 \mathrm{ff}$.

17) DuChASSAING et MichliotTi, Sur les Coralliaires des Antilles. Mem. della R. Acad, d. Torino. II. Tome XIX 1860. S. 292 und 310.

18) KÖLLIKER, Icones Histologicae, II, Abt. 1. Heft. 1865. S. 141. Taf. XII, Fig. 10, 11.

19) KöLLIKER, Vrhdlg. d. phys. med. Gesellsch. Würzburg, N. F. Bd. II. Heft I. S. 12.

20) WRIGHT, Quart. Journ. Micr. Sci. Vol. V. 1865. S. 213-217.

21) VERRILL, Classification of Polyps. Proc. of the Essex Institute. Vol. IV. Salem 1866. S. 148.

22) F. MÜLLER, Archiv für Naturg. Jahrg. XXIII 186\%. S. 56 Anm.

23) VERRILL, Notes on Radiata. Trans. Connecticut Acad. Vol. I, 1868. pt. 2, no 6. S. 455 .

24) VERRILL, Critical Remarks on the Halcyonoid Polyps in the Museum of Yale College. Am. J. Sei. and Arts, Vol. XIV, 1868. S. 415.

25) GRAY, Descriptions of some new Genera and Species of Aleyonoid Corals. Annals and Magazine of Nat. Hist. Vol, III, 4. ser. 1869. S. 21.

26) KENT, Two new Genera of Aleyonoid Corals. Q. J. Mier. Sci. Vol. XVIII, 1870. S. $397, \mathrm{Pl}$. XXI.

27) VERRILL, Radiata from the Coast of North Carolina. Am. J. Sci. and arts, ser. 3, Vol. III, 1872. S. 434.

28) VERRILL, Results of recent Dredging Expeditions on the Coast of New England. Am. J. Sc. and Arts, ser. 3, Vol. VII. 1874. S. 40.

29) KLUNZINGER, Die Koralltiere des roten Meeres, I. Berlin 1877. S. 42.

30) STUDER, Aleyonaria der Gazelle. Monatsber, d. k. preuss. Akad. d. Wiss. Berlin. Okt. 1878. S. 632. Pl. I, Fig. 4.

31) $\mathrm{KOCH}$, Das Skelett der Alcyonarien. Morphol. Jahrb. IV. 1878. S. 447.

32) KOCH, Anatomie der Clavularia prolifera n. sp. Morph. Jahrb. Vol. VII. 1882. S. 467.

33) KOREN og DANIELSSEN, Nye Alcyonider, Gorgonider og Pennatulider tilhörende Norges Fauna. Bergens Museum 1883.

34) KOWALEWSKY et MARION, Documents pour l'histoire embryogénique des Alcyonaires. Ann. du Musée de Marseille. Vol. I. Mémoire 4. 1883.

35) HERDMAN, On the Structure of Sarcodictyon. Proceed. Roy. Soc. Edinb. VIII, 1883. S. $31 \mathrm{ff}$.

36) DANIELSSEN, Norske Nordhars Expedition. Aleyonida 188\%. S. 138.

37) STUDER, Versuch eines Systems der Alcyonaria. Archiv f. Naturg. 53. Jahrg. 1887.

38) KOCH, Die Gorgoniden des Golfes von Neapel. Fauna und Flora des Golfes von Neapel. XV. Berlin $188 \%$.

39) GRIEG, To nye Cornularier fra den Norske kyst. Bergens Museum. 1888. No. 2.

40) WRIGHT and STUDER, Report on the Alcyonaria collected by H. M. S. Challenger. Zool. XXXI. 1889. S. XI und 252.

41) KOCH, Die Alcyonaceen des Golfes von Neapel. Mitteilungen aus der zool. Station Neapel. Vol. IX, 1891. S. 6วั2. 
42) KOCH, Die system. Stellung von Sympodium coralloides. Zool. Jahrb. V. Jena 1891. S. 76 .

43) HICKSON, A Revision of the Genera of the Alcyonaria Stolonifera. Transact. Zool. Soc. Lond. Vol. XIII, Part. 13. 1895.

44) SCHENK, Clavulariiden, Xeniiden und Aleyoniiden von Ternate. Frkft.a.M. 1896. S, 43 ff. In: KÜKENTHAL, Ergebnisse einer zojl. Forschungsreise i. d. Molukken und auf Borneo. Abhandl. der Senkenb. naturf. Gesellschaft. Bd. XXIII, Heft 1. Frkft. a. M. 1896.

Synonyma: Cornulariadae Dana (13).

Cornulariadae KöLL. (18).

Cornularinae + Telestinae M.-E. (15).

Cornularidae VerRILI (21).

Cornularinae KLZGR. (29).

Cornularida v. Косн (31).

Cornulariidae Stud. Wrght. (pars) (40).

Clavulariidae Hıckson (43).

Diagnose: Alcyonaceen, deren Polypen durch kriechende Stolonen oder eine Basalmembran verbunden sind, aus denen die Knospen hervorgehen. Die Basen der Polypen verschiedenen Alters liegen in gleicher Höhe. Polypen daher von nahezu gleicher Länge.

Systematik: Die Familie der Clavulariiden umfaßst gegenwärtig 66 Arten, die sich auf 5 Gattungen verteilen. Doch ist zu beachten, daf3 ein großer Teil dieser Arten nur sehr unvollkommen bekannt ist und die Zahl sich bei einer gründlichen Revision der ganzen Familie wahrscheinlich sehr beträchtlich reduzieren wird. Die wichtigsten Charaktere der Gattungen ergeben sich aus folgender Übersicht:

I. Polypen niemals vollständig in die Stolonen oder die Basalmembran zurückziehbar :

1. Hornscheide vorhanden. Spicula fehlen:

Cornularia Lm.

2. Hornscheide fehlt. Spicula meist vorhanden:

Clavularia Q. G.

II. Polypen vollständig in die dicke Basalmembran zurückziehbar:

Sympodium Ehrbg.

Anthopodium Verrill

Callipodium Verrill.

\section{Litteratur:}

\section{Gattung: Clavularia Q. G.}

Siehe Clavulariidae.

Synonyma: Clavularia Q. G. + Anthelia LM. +- Rhizoxenia Eнrвg. +- Sarcodictyon Forb. + Gymnosarca Kent + Cornulariella VerRILL + Stercosoma Hickson. 
Diagnose: Clavulariiden mit Stolonen oder Basalnuembran, in die die Polypen sich nicht vollstïndig zurückziehen können. Spicula meist vorhanden. Hornscheide fehlt.

Man kennt bis jetzt 40 Arten der Gattung Clavularia, von denen Hıckson (43) eine übersichtiiche Darstellung gegeben hat. Dazu kommen die in Folgendem beschriebenen 3 neuen Arten.

\section{Clavnlaria longissima n. sp.}

Diagnose: Pinnulae in einer Reihe jederseits der Mittellinie der Tentakeln, lang, wurstförmig. Kalkkörper fehlen.

Beschreibung: Von einer zarten membranösen Basis, die einen Madreporenzweig überzieht, erheben sich zahlreiche langgestreckte Polypen, die sich mit ihren Basalenden berühren. Ihre Länge beträgt ohne die Tentakeln 15 bis $54 \mathrm{~mm}$. Ein Teil der Polypen ist cylindrisch, in der ganzen Lünge bis $2 \mathrm{~mm}$ breit, ein anderer Teil ist an der Basis und an der Spitze mehr oder weniger stark blasenförmig angeschwollen, ein dritter Teil zeigt die Anschwellung nur an der Spitze, ein rierter nur an der Basis. Die obere Blase ist entweder kugelig, und die Tentakeln sitzen dann dichtgedrängt auf dem obern Endpunkt des senkrechten Durchmessers; oder sie ist halbkugelig, in welchem Fall die Tentakeln rings um die kreisförmige Mundscheibe angeordnet sind. Die Tentakeln werden bis $12 \mathrm{~mm}$ lang, haben eine cylindrische, nach oben allmählich sich zuspitzende, im mittleren Teil etwa $0,348 \mathrm{~mm}$ lange Axe und jederseits eine Reihe von langen wurstförmigen, nach aufwärts gekrümmten Pinnulae. Kalkkörper fehlen. - Zwei weitere Exemplare weisen keine besondern von der beschriebenen Form abweichende Eigentïmlichkeiten auf. Die Farbe der Kolonieen ist hellbraun.

Fundort: Kokatoni. 3 gut erhaltene Exemplare.

\section{Clavularia gracilis $\mathrm{n}$. sp.}

Diagnose: Pinnulae in 3 unregelmäßigen Reihen jederseits der T'entakelaxe, kurz und dick; auf jeder Fläche des Tentakels bleibt meist nur ein schmaler Teil frei. Kalkkörper fehlen. Polypen sehr schlank, oft am obern Ende trichterförmig erweitert.

Beschreibung: Eine dünne, hüutige, $70 \mathrm{~mm}$ lange Basalme $\mathrm{mbran}$ überzieht einen Laminarienstiel. Ringsum erheben sich von ihr zahlreiche Polypen in Entfernungen von 1-2 mm und von sehr verschiedener Länge. 1)e kürzesten sind weniger als $1 \mathrm{~mm}$, die lïngsten $12 \mathrm{~mm}$ lang. Die Breite ist nach dem Kontraktions\%ustand verschieden, ganz ausgestreckte Polypen sind nur $0,46 \mathrm{~mm}$ dick, aber am obern Ende trichterförmig erweitert. I ie Tentakeln sind bis $5 \mathrm{~mm}$ lang und im mittlern Teil mit den P'inuulae nicht granz (1,5 $\mathrm{mm}$ breit. Die P'innulae sind kurze und dicke 
Wärzchen, die jederseits der Tentakelaxe in 3 unregelmäßigen Reihen stehen; auf beiden Seiten bleibt meist nur ein schmaler Teil der Axe frei. Die F arbe der Kolonie ist hellbraun. - Ein zweites Exemplar hat einen Schwamm umwachsen, ein drittes umfasst einen Madreporenast.

Fundort: Tumbatu.

\section{Clavularia flava 1 . sp.}

Diagnose: Pinnulae in 4 Reihen auf jeder Seite der Tentakeln, kurz, dick und stumpf. Tentakelaxe in ihrer ganzen Länge frei. Kalkkörper ovale gelbbraune Scheiben von 0,02 mm Länge und 0,012 mm Breite.

Beschreibung: Die Kolonie ist mit der Basalmembran auf einem Schwamm aufgewachsen. Die Polypen sind bis $10 \mathrm{~mm}$ lang und $1 \mathrm{~mm}$ breit. Ihre Basalenden berühren sich oder sind nur durch kleine Zwischenräume von einander getrennt. Die Tentakeln sind bis $6 \mathrm{~mm}$ lang, an der Basis bis $1 \mathrm{~mm}$ breit, von lanzettförmiger Gestalt. Die Pinnulae stehen in 4 Reihen auf jeder Seite der Tentakeln; sie sind kurz, dick und meist stumpf und lassen die Tentakelaxe in ihrer ganzen Länge frei. Die Kalkkörper sind ovale gelbbraune Scheiben von $0,02 \mathrm{~mm}$ Länge und $0,012 \mathrm{~mm}$ Breite. Sie sind in großer Zahl vorhanden. Die F arbe der Kolonie ist hellgelblich.

Fundort: Sansibar, 3 Bruchstïcke.

\section{Clavularia celebensis Hickson.}

Litteratur:

1) HICKSON, A Revision of the Genera of the Alcyonaria Stolonifera. Transact. Zool. Soc. Lond. Vol. XIII, Part. 13, 1895. S. 342. Pl. XLVIII.

Diagnose: Pinnulae in 4 Reihen jederseits der Mittellinie der Tentakeln, ziemlich lang. Kalkkörper fehlen.

Beschreibung: Diese Spezies wurde zuerst 1895 von Hickson (1) beschrieben und gut abgebildet. Das mir vorliegende Exemplar unterscheidet sich von der von Hickson beschriebenen Form durch die geringere Dicke der Polypenwände und die Basalmembran. Die B as a l me m bran überzieht einen Schwamm. Die Polypen sind sehr zahlreich und gruppenweise angeordnet. Ihre Länge schwankt zwischen 1 und $15 \mathrm{~mm}$. Die Breite beträgt bis $2 \mathrm{~mm}$. Die Tentakeln sind zungenförmig, bis $5 \mathrm{~mm}$ lang und an der Basis bis $0,794 \mathrm{~mm}$ breit. Die ziemlich langen Pinnulae stehen in 4 Reihen auf jeder Seite der Tentakeln. Kalkkörper fehlen. Die Farbe der Kolonie ist hellbraun.

Fundort: Tumbatu, 1 Exemplar.

Litteratur:

\section{Gattung: Sympodium Ehrbg.}

1) PALLAS, Elenchus Zoophytorum. Haag 1766. S. 192.

2) FORSKAL, Descriptiones animalium, quae in itinere orientali olservavit, 1775. S. 139. 
8) FORSKAL, Ieones rerum naturakium, 1776. Taf. $37 \mathrm{~A}$, a.

4) O. FR. MÖLLER, Zoologia Danica. Hafniae 1779-80. Vol. III, S. 2. Pl. 82, Fig. 1-4.

5) ELLIS and SOLANIFE, The natural history of many curious and uncommun Zoophytes. London 1786, S. 181. Taf. 9, Fig. 1, 2.

6) EsP'ER, Die Pflanzentiere. Nürnberg 1791-97. II. Teil, S. 59 u. 108. Gorgonien, Taf. 14 u. 32.

7) SAVIGNY, Description de l'Egypte. Hist. nat. Tome I, 4. partie. Polypes, Pl. I, Fig. 4, 6, 7 .

8) BLainville, Manuel d'Actinologie. Paris 1834, S. 683.

9) EHRENBERG, Die Korallentiere des roten Meeres. Berlin 1834, S. 61.

10) DANA, Zoophytes, Philadelphia 1846, S. 608.

11) MILNE-EDWARDS, Hist. nat. d. Coralliaires. Tome I, Paris 1857, S. 110.

12) DANA, Synopsis, New Haven 1859, S. 122.

13) DICHASSAING et MICHELOTTI, Mémoire sur les coralliaires des Antilles. Torino 1860 , S. $290,292$.

14) KöLLIKER, Icones histologicae, T. II, Leipzig 1865, S. 141. Taf. XIX, Fig. 7-9.

15) VERRILI, Proc. Boston S. N. H. 1866.

16) GRAY, Notes on the Fleshy Alcyonoid Corals. Ann. and Mag. of Nat. Hist. London 1869, S. 119 u. 120.

17) KLUNZINGER, Die Koralltiere des roten Meeres. Teil I, Berlin 1877, S. 42, Taf. III, Fig. 5-8.

18) KOREN og DANIELSSEN, Nye Alcyonider, Gorgonider og Pennatulider. Bergen 1883. S. 14.

19) GRIEG, Bidrag til de norske alcyonarier. Bergens Museums Aarsberetning for 1886, S. 3.

20) DANIFLSSEN, Norske Nordhavsexpedition, Alcyonida, Kristiania 1887, S. 141, Taf. 23.

21) STUDER, Versuch eines Systems der Alcyonaria. Archiv f. Naturg. 53 Jahrg. I. Bd. Berlin 1887, S. 4, 11 ษ. 12.

22) WRIGHT and STUDER, Report on the Alcyonaria coll. by H. M. S. Challenger. Chall. Report, Bd. XXXI, 1889, S. XV. u. 270 ff. Taf. 42 u. 43.

23) KOCH, Die systematische Stellung von Sympodium coralloides Pall. Zool. Jahrb. V. Bd. 1891, S. 76 .

24) HICKSON, A Revision of the Genera of the Alcyonaria Stolonifera. Trans. of the Zool. Soc. of Lond. 1895, S. 336.

Synonyma: Anthelia Sav. (7) (pars).

Massarella + Eunoella + Sympodium + Erythropodium + Ojeda Gr. (16).

Diagnose: Auf einer dicken membranösen, von Ernährungskanälen durchzogenen Basis, die fremde Körper !berzieht, erheben sich kurze mehr oder weniger vollkommen retraktile Polypen. Spicula sehr verschieden.

Die Zahl der bis jetzt bekannten Sympodium-Arten betrïgt 18. Davon sind einige aber sehr ungenügend charakterisiert.

\section{Litteratur:}

\section{Sympodium coeruleum Ehrbg.}

1) SAVIGNY, Description de l'Egypte. Hist. nat. Pl. T. II, Paris 1817, Polypes, Pl. I, Fig. 4.

2) EHRENBERG, Die Kurallentiere des roten Meeres, Berlin 1834, S. 61. 
3) DANA, Zoophytes, Philadelphia 1846, S. 609.

4) MILNE-EDWARDS, Hist. nat. des Coralliaires. T. I, Paris 1857, S. 111.

5) DANA, Synopsis, New-Haven 1859, \$. 122.

6) GRAY, Notes on the fleshy alcyonoid corals. Ann. and Mag. of Nat. Hist. Vol. III, 4. ser. 1869, S. 120.

7) KLUNZINGER, Die. Koralltiere des roten Meeres, Teil I, Berlin 1877, S. 42.

8) WRIGHT u. STUDER, Report on the Alcyonaria collected by H. M. S. Challenger. Chall. Report Bd. XXXI, 1889, S. 271.

Diagnose: Basalausbreitung flach. Polypen 3-4 mm lang. Kalkkörper mikroskopisch klein, scheibenförmig. Farbe hellblau.

Historisches: Ehrenberg (2) giebt 1834 folgende Diagnose dieser Art: „Effusum, obducens, membrana tubulisque fuliginosis, tentaculis laete caeruleis, parvis, gracilibus". KLUNZINGER (7) veröffentlicht 1877 die Ehrenberg'schen Abbildungen und liefert eine genauere Beschreibung. Er identifiziert die Spezies mit der von SAvigry (1) auf Tafel I, Fig. 4 abgebildeten Form. Mir liegen einige kleine Kolonieen aus Tumbatu vor, die auf abgestorbenen Madreporenästen aufgewachsen sind. Sie haben durchaus den Charakter von Cornulariden. Die Polypen sind alle ausgestreckt.

Litteratur:

\section{Sympodium fulvum (Forsk.)}

1) FORSKAL, Descriptiones animalium 1775, S. 139.

2) FORSKAL, Icones rerum naturalium 1776 , Taf. $37 \mathbf{A}$, a.

3) EIRENBERG, Die Koraltentiere des roten Meeres, Berlin 1834, S. 156.

4) KLUNZINGER, Die Koralltiere des roten Meeres, Teil I, Berlin 1877, S. 43, Taf. III, Fig. 6.

5) WRIGIIT und STLIDER, Report on the Alcyonaria collected by I. M. S. Challenger. Chall. Report Bd. XXXI, 1889, S. 271.

Synonyma: Lithophyton fulvum Forsk. (1 u. 2). Sympodium fulvum EнRвG. (3).

Diagnose: Basalausbreitung oben hügelig. Polypen $2-3 \mathrm{~mm}$ lang. Kalkkörper des Coenenchyms mit bloßem Auge deutlich sichtbar, spindelförmig, mit sehr feinen Warzen besetzt (fast glatt). Spicula der Polypen von derselben Gestalt, die untern horizontal, die darüber liegenden in S Doppelreihen angeordnet. Farbe dunkelgelb.

Historisches: Diese Art wurde bereits Ende des vorigen Jahrhunderts von ForskaL ( 1 u. 2) als Lithopliyton fulvum beschrieben und abgebildet. Ehrenberg (3) stellt sie 1834 mit folgender Diagnose zur Gattung Sympodium: „Effusum, obducens, aureofulvum, membranaceum, tenue“. Line ausführlichere Beschreibung mit Abbildung giebt KLunzinger (4) 18\%\%. Ich habe ihr nur hinzuzufügen, dał die spindelförmigen Spicula mit ganz feinen Warzen besetzt sind. Trotz der auf der obern Seite der Hach ausgebreiteten Kolonie sich erhebenden Hügel muß die Spezies als eine Cornularide aufgefalist werden, da alle Polypen bis zur Basis herab- 
gehen und nicht wie bei den Alcyoniden in verschiedener Höhe über ihr entspringen. Die mir vorliegenden Exemplare sind aus $\mathrm{T} u \mathrm{mb}$ atu und teils auf abgestorbenen Madreporenästen, teils auf Laminarienstielen aufgewachsen, die sie rings umgeben. An der Spitze der Äste setzen sie sich noch eine Strecke weit tort, aber auch hier konnte ich die für die Alcyoniiden charakteristische Anordnung der Polypen nicht entdecken.

\section{Sympodium punctatum n. sp.}

Diagnose: Kalkkörper farblose oder intensiv rote, grade oder gebogene Stäbe, die mit längern und kürzern bedornten Warzon unregelmäßig besetzt sind. Die roten Spicula bilden eine besondere tiefere Schicht im Coenenchym.

Beschreibung: Die Kolonieen überziehen Laminarienstiele. Die Polypen sind vollständig eingezogen und erscheinen infolge ihrer rotgefärbten Tentakeln als rote Punkte auf dem grauen Coenenchym. Die der Unterlage unmittelbar aufliegende tiefste Schicht des Coenenchyms ist durch die roten Spicula rot gefürbt. Die Spicula sind von denen der vorhergehenden Spezies total verschieden und nähern sich etwas denen von Alcyonium coralloides (PALs.), so daf die Vermutung nahe liegt, daf wir es hier ebenfalls mit einer Alcyoniide zu thun haben. Die mir vorliegenden Lxemplare sind aber zu schlecht erhalten, um die Frage mit Sicherheit entscheiden zu können. Die Kalkkörper sind verästelte Stäbe. Die größten sind $0,266 \mathrm{~mm}$ lang.

Fundort: Tumbatu, S. W. Riff.

\section{Familie: Telestidae (M.-E.).}

\section{Litteratur:}

1) MILNE-EIWARDS, Histoire naturelle des Coralliaires. Tome I. Paris 185\%. S. 112.

2) KOCH, Anatomie der Clavularia prolifera. Morph. Jahrb. Bd. VII, 1881. S. 481.

3) KOCH, IDie Gorgoniden des Golfes von Neapel. Fauna und Flora des Golfes von Neapel. XV. Monographie, Berlin 188\%, S. 6, 9 und 15.

4) HI('KSON, A Revision of the Genera of the Alcyonaria Stolonifera. Transactions of the Zoological Society of London. Vol. XIII. London 1895. S. 334.

Synonyma: Telestinae M.-E. (1).

Diagnose: Alcyonaceen, deren Kolonien aus aufrechten Axenpolypen und lateralen Polypen zweiter bis dritter Ordnung bestehen. I)ie I)armhöhlen der lateralen Polypen stehen durch mesodermale Stolonen in der verdickten Wand der Mutterpolypen mit deren Darmhöhlen in Verbindung. Die Stolonen sind also mit ihrem Ursprung nicht auf die Basis der P'olypen beschränkt, sondern gehen von verschiedenen Punkten der Ideibeswand aus.

Systematik: Man kennt bis jetzt 13 Arten der Telestiden, die sich auf 4 Gattungen verteilen. I)ie Charaktere der (rattungen ergeben sich aus folgender Übersicht: 
I. Kolonieen mit Polypen erster und zweiter Ordnung. Polypen retraktil:

A. Axialpolyp ohne sterilen Teil.

1. Laterale Knospung undeutlich:

Scleranthelia Tн. STоD.

2. Laterale Knospung deutlich:

Telesto Lax.

B. Axialpolyp mit sterilem Teil:

Pseudogorgia KöLL.

II. Kolonieen mit Polypen erster, zweiter und dritter Ordnung. Polypen nicht retraktil.

Coelogorgia M.-E.

Litteratur:

Gattung: Coelogorgia M.-E.

1) VAleincienneS, Mss. Coll. du Mus. Jard. des Plantes. Paris.

2) MILNE-EDWARDS, Hist. nat: des Coralliaires, Tome I, Paris 1857, S. 191.

3) VERRILL, Mem. Bost. Soc. Vol. I, S. 5, 1866.

4) WRIGHT and STUDER, Report on the Alcyonaria collected by H. M. S. Challenger. Chall. Rep., Zool. Vol. XXXI. S. XIV und 265.

Synonyma: Lobularia SAv. (pars) (1).

Diagnose: Kolonie baumförmig. Stamm durch einen Axialpolypen gebildet, von dessen Körperwand zweigartige Polypen zweiter Ordnung entspringen, die wieder Polypen dritter Ordnung tragen. Die zweigartigen Polypen zweiter Ordnung können auch noch einmal Zweigen den Ursprung geben, an denen dann Polypen vierter Ordnung sitzen. Polypen nicht retraktil. Spicula spindelförmig.

\section{Coelogorgia palmosa (Val.).}

Litteratur:

Siehe Coelogorgia.

Synonyma: Lobularia palmosa Vat. (1).

Diagnose: Siehe Genusdiagnose.

Beschreibung: Wright und Studer (4) haben von dieser Art eine sehr gute und ausführliche Beschreibung gegeben, der ich nichts hinzuzufügen habe. In meinem Material befinden sich mehrere Stämme, von denen der größte $220 \mathrm{~mm}$ hoch ist.

Fund ort: Sansibar, Tumbatu.

Litteratur:

\section{Familie: Tubiporidae Gray.}

1) LINNÉ, Systema naturae. Edit. X. Tome I. 1758. S. 789.

2) PALLAS, Elenchus Zoophytorum. 1766. S. 339.

3) ELLIS und SOLANDER, Natural History of Zoophytes. 1786. Taf. 27, 1 und 2.

4) LAMARCK, Hist. nat. des anim. s. vert. Tome II. Paris 1816. S. $20 \%$. 
5) 1.AMothoux, Expos. méth. des Genres de l'ordre des Polypiers. Paris 1821. S. 66, Taf. $2 \%$.

6) (g)OY et GaIMARI), Yoyage in l'Astrulabe, Zoul. Paris 1833. S. 25\%. Pl. 21, Fig. 1-8.

7) EHRENBEHG, Die Korallentiere des roten Meeres. Berlin 1834. \$. 55.

8) BLAinville, Manuel d'Áctinologie. Pris 1834. S. 500.

9) DANA, Zoophytes. Pliladelphia 1846. S. 633.

10) MLLNE-LIWARDS, Hist. nat. des coralliaires. Tome I. Paris 185\%. S. 130.

11) DANA, Synopsis. New Haven 1859. S. 127.

12) GRAY, On the Arrangement of Zoophytes with Pinnated Tentacles. Ann. and Mag. of Nat. Hist. Vol. IV, 3. ser. Lond. 1859. S. 444.

13) KöLLIKER, Icones Histiologicae. Abt. II. Heft I. Leipzig 1865. S. $16 \%$.

14) VERRILL, Classification of Polyps. Proceed. of the Essex Institute Vol. IV. Salem 1866. S. 148.

15) WRIGHT, Notes on the Animal of the Organ-pipe Coral (Tubipora musica). Ann. and Mag. of Nat. Hist. Vol. III, 4. ser. Lond. 1869. S. $37 \%$.

16) KO('H, Anatomie der Orgelkoralle (Tubipora Hemprichii Ehrbg.). Jena 1874.

17) KLUNZiNGER, Die Koralltiere des roten Meeres. Teil I. Berlin 1877. S. 46.

18) STUDER, Alcyonarien der Gazelle. Monatsber. d. kgl. Akad. d. Wiss. z. Berl. 1878. S. 634 .

19) KOCH, Das Skelett der Alcyonarien. Morph. Jahrb. Bd. 1V. 1878. \$. $474 \mathrm{ff}$.

20) CARTER, Report on Specimens dredged up from the Gulf of Manaar. Ann. and Mag. of Nat. Hist. 5. ser. Vol. V. 1880. S. 442.

21) HICKSON, On the Ciliated Groove (Siphonoglyphe) in the Stomodaeum of Aleyonariens. Proc. Royal Soc. No. 226. 1883.

22) HICKSON, The Strueture and Relations of Tubipora. Quart. Journ. Micr. Sci. 1883. S. $516-528$.

Synonyma: Tubiporina Errib. (7).

Tubiporidae Dana (pars) (9).

Tubiporinae M.-E. (10).

Tubiporidae Gr. (12).

Tubiporidae VerRiLl (14).

Tubiporida Косн (19).

I)iagnose: Spicula zu Kalkröhren verschmolzen, die in größerer Zahl parallel neben einander stehen und in verschiedener Höhe durch horizontale Stolonenplatten verbunden sind, aus denen die Polypen entspringen. Vorderer Teil der Polypen retraktil.

Systematik: Die Familie der Tubiporiden umfaßst nur eine Gattung, und in dieser hat man bis jetzt 8 Arten unterschieden. Als Speciescharaktere betrachtet man: 1) die Zahl der P'innulaereihen auf den Tentakeln, 2) die Entfernung der Polypenröhren von einander, 3) die Entfernung der.Querbrücken von einander, 4) die Entfernung der Tabulae von einander, 5) die Gestalt der Tabulae, 6) die Weite der Röhren, 7) die Farbe der Polypen. Bei der außserordentlichen Relativität dieser Charaktere ist die Bestimmung, besonders bei Spiritusexemplaren schwierig und unsicher. 
Litteratur:

\section{Gattung: Tubipora $L$.}

Siehe Tubiporiden.

Diagnose: siehe Tubiporiden.

\section{Tubipora rubeola $Q$. G.}

Litteratur:

var. sansibarica nov.

1) QUOY et GAYMARD, Voyage de découvertes de l'Astrolabe. Zoologie. Paris 1833. S. 257, Pl. 21, Fig. 1-8.

2) DANA, Zoophytes. Philadelphia 1846. S. 636.

3) MILNE-EDWARDS, Hist. nat. des Cor. 'Tome I. Paris 185\%. S. 133.

4) DANA, Synopsis. New Haven 1859. S. 128.

5) STUDEk, Alcyonarien der Gazelle. Monatsber. der königl. Akad. der Wiss. zu Berlin, 1878. S. 634 .

Diagnose der Stammform: Pinnulae in 2 Reihen auf jeder Seite der Tentakeln. Polypenröhren entfernt, Stolonenplatten spärlich, Röhren weit, Tentakeln rot.

Von dieser Diagnose der Stammform unterscheidet sich das mir vorliegende Exemplar hauptsächlich dadurch, daß die Stolonenplatten zahlreich sind und in geringen Entfernungen übereinander stehen. Ich habe daher eine Varietät aufgestellt. Die Polypenröhren sind gegen $2 \mathrm{~cm}$ weit.

Fundort: Sansibar.

\section{Tubipora chamissonis Ehrbg.}

\section{Litteratur:}

1) CHAMISSO et EISENHARDT, De animalibus quibusdam e classe vermium Linnaeana etc. Nov. Act. Caes. Leop. T. X, 1821.

2) FREYCINET, Voyage autour du monde sur les Correttes l'Uranie et la Physicienne. Paris 1824.

3) EHRENBERG, Die Korallentiere des roten Meeres. Berlin 1833. S. 56.

4) DANA, Zoophytes. Philadelphia 1846. S. 635.

5) MILNE-EDWARDS, Hist. nat. des Cor. Tom. I. Paris 185\%. S. 133.

6) DANA, Synopsis. New Haven 1859. S. 128.

Synonyma: Tubipora musica CHАM. (1).

Diagnose: Pinnulae in 2 Reihen auf jeder Seite der Tentakeln. Polypenröhren dichtstehend, Stolonenplatten zahlreich, Polypenröhren etwa $2 \mathrm{~mm}$ weit.

Historisches: Diese Art ist zuerst von Chamisso (1) auf der ersten Reise KотzевеE's beobachtet, in dem 1821 herausgegebenen Reisewerk abgebildet und als T. musica beschrieben worden. Später gaben Quor und Gaimard in dem Reisewerk Freycinet's (2) über die Weltumseglung der Uranie eine neue Abbildung. Ehrenberg (3) bezeichnet die Art 1833 als T. chamissonis und giebt ihr die Diagnose: "Semipedalis, laete rubra, tubis 
3o" latis, densius confertis, dissengmentis crebrioribus, animalis tontaculis dupliciter pinnatis. In linea polliciri superticies $10-15$ tubulos offert." - Die mir vorliegenden Exemplare sind aus Sansibar.

\section{Familie: Xeniidae Verrill.}

\section{Litteratur:}

1) LSPER, Die Pflanzentiere. Nürnberg, 1791-179\%. Bd. 3, S. 20 u. 49. Taf. III u. XVI.

2) LAMARCK, Hist. nat. des animaux s. vert. T. II, Paris 1816. S. $403 \mathrm{ff}$.

3) SAVIGNY, Description de l'Egypte. Hist. nat. T. I, 4. partie. Paris (ohne Jahresangabe), S. 227 ff. Planches T. II. Paris 1817, Polypes Pl. I, Fig. 3.

4) SCHWEIGGER, Beob. auf naturhist. Reisen. Berlin 1819. S. $94 \mathrm{ff}$.

5) LAMOUROUX, Exposition méthodique des genres de l'ordre des polypiers. Paris 1821, S. 69.

6) LLSSON, Voyage de la Coquille. Zooph. 1825. S. 85. Pl. I. Fig. 3.

7) QUOY et GAIMARD, Voyage de découvertes de l'Astrolabe. Zoologie. T. IV. Paris 1833. S. 265 ff. Planche 22, Fig. 1-\%.

8) EHRENBERG, Die Korallentiere des roten Meeres. Berlin 1834. S. 53 ff.

9) BIAINVILLE, Manuel d'Actinologie, P’aris 1834, S. 523 und 682. Pl. $88 \mathrm{I}$, Fig. 5.

10) TEMPLETON, Trans of the zool. soc. of London, 1841, Vol. II, S. 25, Fig. 3-7.

11) DANA, Zoophytes, Philadelphia 1846. S. 604 ff. Pl. 57, Fig. 4 u. 5.

12) MILNE-EDWARDS, Hist. nat. des Coralliaires. T. I. Paris $185 \%$ S. 113 u. $125 \mathrm{ff}$.

13) DANA, Synopsis of the Report on Zonphytes. New-Haven 18ă9. S. $119 \mathrm{ff}$.

14) GRAY, On the arrangement of Zoophytes with pinnated tentacles. Annals and Magazine of Nat. Hist. Vol. IV. 3. ser. Lond. 1859. S. 443.

15) SARS, Bidrag til Kundskaben om Middelhavets Littoralfauna. S. 4.

16) DUCHASSAING et MICHELOTTI, Mém. sur les coralliaires des Antilles. Torino 1860 . S. $292 \mathrm{ff}$. Taf. I, Fig. 1 u. 2, 8 bis 11.

17) KöLLIKER, Icones Histologicae II, 1. Heft. Leipzig 1865. S. 133.

18) VERRILL, Classification of Polyps. Proc. of the Essex Inst. Vol. IV. 1864-65. Salem 1866. S. 148.

19) GRAY, Notes on the fleshy Alcyonoid Corals. Annals and Magazine of Nat. Hist. Vol. III. 4. Ser. Lond. 1869. S. 126.

20) KöLLIKER, Die Pennatulide [nubellula und zwei neue Typen der Aleyonarien. Festschrift zur Feier des 2õ jähr. Best. d. phys. med. Gesellsch. i. Würzburg. Würzburg 1874, S. 12 ff.

21) KLTXZINGER, Die Koralltiere des roten Meeres, Teil I. Berlin 18\%7. S. อิ 9 ff.

22) STUDER, Übersicht der Anthozoa Alcyonaria, welche während d. Reise S. M. S. Gazelle gesammelt wurden. Monatsber. d. Königl. Akad. d. Wiss. z. Berlin. Okt. 1878. S. 633.

23) HAACKE, Zur Physiologie der Anthozoen. Der Zool. Garten. XXVII. Jahrg. Frkft. a, M. 1886。 S. 284 ff。

24) STLDER, Versuch eines Systems der Alcyonaria. Arch. f. Naturg. 53. Jahrg. I. Band. Berlin 1887. S. 14

25) WRIGHT and STLDER. Report on the Alcyonaria collected by H. M. S. ('hall. during the years 1873-76. Report on the scientific results of the voyage of H M. S. Cball. Zool. Vol. XXXI, 1889。 S. XVII u. 252.

26) HICKSON, A Revision of the Genera of the Alcyonaria Stolonifera. Trans. Zool. Society. Lond. Vol. XIII, Part, 13. 1895. 
27) BRUNDIN, Alcyon. a. d. Samml. d. zool. Mus. z. Upsala. Bihang till Svenska Vet. Akad. Handlingar. Bd. 22. Afd. IV, No. 3. Stockh. 1896. S. 4.

28) SCHENK, Clavulariiden, Xeniiden u. Aleyoniiden von Ternate. S. $50 \mathrm{ff}$. In KÜKENTHAL, Ergebnisse einer zool. Forschungsreise i. d. Molukken u. in Borneo. Frkft. a. M. 1896.

Synonyma: Polypi tubiferi LM. (pars) (2).

Xenina Errbg. (pars) (8).

Xeninae Dana (pars) (11 u. 13).

Alcyoninae M.-E. (pars) (12).

Xeniadae Gr. (pars) (14 u. 19).

Xeniidae Verrilu (18).

Alcyoninae exsertae KLzGR. (pars) (21).

Xeniidae Th. STud. (24).

Xeniidae Stud. Wraht. (25).

Xeniidaie Schenk (28).

Diagnose: Alcyonaceen; deren zu Bündeln vereinte Polypen im untern Teil durch Coenenchym verbunden sind, das einen zuweilen verzweigten Stiel bildet, auf dessen oberer Fläche der freie Teil der Polypen sich doldenartig erhebt. Polypen nicht retraktil. Coenenchymmasse mit Kanalsystem.

Zahl der Arten: Bis jetzt sind 25 Xeniidenarten bekannt, die sich auf 2 Gattungen Xenia und Cespitularia verteilen.

\section{Gattung: Xenia Sav.}

Litteratur:

Siehe Xeniiden.

Synonyma: Actinantha Lessor (6).

Heteroxenia KöLı. (20).

Diagnose: Xeniiden, bei denen der sterile Strunk und die fertile Endscheibe deutlich von einander abgegrenzt sind.

Systematisches: Man kennt bis jetzt 20 Arten der Gattung Xenia. Von diesen waren 8 in dem von mir untersuchten Material vorhanden, darunter 4 neue.

Litteratur:

\section{Xenia nmbellata Sav.}

1) LAMARCK, Hist. nat. des animaux sans vertèbres. Tome II. Paris 1816. S. 410.

2) SAVIGNY, Description de l'Egypte. Hist. nat. T. 1. 4. partie. Paris. S. $22 \%$. Pl. T. II. Paris 1817, Polypes Pl. I, Fig. 3.

3) SCHW'EIGGER, Beob. auf naturhist. Reisen. Berlin 1819. S. 94. Taf. V, Fig. 48-50.

4) LAMOUROUX, Expos. méth. des genres de l'ordre des Polypiers. Paris 1821. S. 69.

5) EHRENBERG, Die Korallentiere des roten Meeres. Berlin 1834. S. 53.

6) BLAINVILLE, Manuel d'Actinologie. Paris 1834. S. 523.

7) DANA, Zoophytes. Philadelphia 1846.

8) MILNE-EDWARDS, Hist. nat. des Coralliaires. Paris 185\% S. 125.

9) DANA, Synopsis, New Haven 1859. S. 119. 
10) KöLLIKER, Icones Histologicae. Leipzig 1865. S. 133.

11) GHAY, Notes on the Fleshy Alcyonoid Corals. The Annals and Magarine of Nat. Ilist. Vol. III, 4. ser. London 1869. S. 126.

12) KólLLKFR, Die Pennatulide Lmbellula ete. Festschrift zur Feier des 25 jähr. Bestehens der Physik. Mediz. Gesellsch. i. Würzburg. Würzburg 1874. S. 1\%.

13) HAECKEL, Arabische Korallen, Berlin 1876. S. 44. Taf. I, Fig. 8.

14) KLAXZINGEli, Die Koralltiere des roten Meeres, T. I. Berlin 1877. S. 39. Taf. III, Fig. 3.

15) SCHFXK, Clavulariiden, Xeniiden u. Alcyoniiden von Ternate. Frkft. a. M. 1896. S.5\%.

Synonyma: Xenia coerulea EHRBg. ist mit Xenia umbellata Sav. zu rereinigen.

Diagnose: Pinnulae in 3 Reihen auf jeder Seite der Mittellinie der Tentakehn, sehr lang, schlink und spitz, nur die tiefst stehenden (sehr wenige) warzenförmig. Axe der 'Tentakeln zungenfömmig, in ihrer ganzen Länge frei.

Historis ches: Xenia umbellata ist rie am lïngsten bekannte Xeniide. I.AMARCK (1) beschrieb sie zuerst 1816 nach den Heobachtungen Savigny's (2), dessen Abbildung im folgenden Jahr veröffentlicht wurde. Beide Forscher geben 2 P'immulacreihen als charakteristisch an. 1819 giebt ScHweigger (3) auf Grund von Untersuchungen der Fixemplare des Hurter'schen Museums zu London eine neue Beschreibung und eine ganz gute Abbildung, auf der deutlich 3 Pinnulaereihen sichtbar sind. I)amit stimmt die neue Diagnose überein, die Fanenbeng (5) im Jahre 1834 giebt und die sich in der Litteratur eingebürgert hat. KLtinzinger (14) und Schenk (15) geben 3-4 Reihen an, damit wird aber die Grenze zwischen Xenia umbellat und Xenia fuscescens verwischt.

Beschreibung: Bei einem der vorliegenden kxemplare wheben sich ron einer membranösen Basis, die einen Madreporarienast ïberzieht, 9 teils cylindrische, teils schwach liegelfürmige Aeste von $15-30 \mathrm{~mm}$ Höhe. Einer der Äste ist an der Basis $6 \mathrm{~mm}$, an der Spitze $11 \mathrm{~mm}$ dick, andere sind oben fast ebenso breit wie unten. Die Oberflïche der Ïste ist glatt. Ein zweites Exemplar ist ein einzelner auf einem kleinen Ästchen aufgewachsener Stamm, $25 \mathrm{~mm}$ hoch, $17 \mathrm{~mm}$ breit und $9 \mathrm{~mm}$ dick. Die Polypen stehen dichtgedrängt auf der Endscheibe des Strunks und werden bis $19 \mathrm{~mm}$ lang und $1 \mathrm{~mm}$ breit. Die Tentakeln sind zungenförmig, bis $5 \mathrm{~mm}$ lang und an der Basis $1 \mathrm{~mm}$ breit. Die untersten Pinnulae sind warzenförmig, die obern schlauchförmig, sehr lang, schlank und spit\%. Sie stehen in 3 Reihen auf jeder Seite der Mittellinie der Tentakeln und lassen die Tentakelaxe frei. Der obere 'Teil der Axe ist zwar von den sich nach innen zu überlegenden Tentakeln verdeckt, die Insertionen de'r Pinnulae erstrecken sich aber nicht bis auf die Mittellinie. Pol y penknospen sind bald sehr zahlreich vorhanden, bald fehlen sie ganz. Die Kalkkörper sind von der gewöhnlichen Forın und Grösse; in den Exemplaren 
meines Materials sind sie selı spärlich, während sie bei den Enrenberg'schen Exemplaren des Berliner Museums, die mir zur Untersuchung zur Verfügung standen, bis in die Spitzen der Pinnulae sehr zahlreich vorkommen. Die Farbe des Stammes und der Polypenkelche ist gelblichweiß, die der Tentakeln etwas dunkler.

Fundort: Tumbatu, Südriff; Insel Baui. 4 Exemplare.

\section{Xenia tumbatuana n. sp.}

Diagnose: Pinnulae in 3 Reihen zu beiden Seiten der Mittellinie der Tentakeln, dick und stumpf, locker angeordnet. Axe der Tentakeln sehr schlank, cylindrisch, nicht zungenförmig, in ihrer ganzen Länge frei.

Beschreibung: Von einer gemeinsamen Basis, die einen Stein umwachsen hat, erheben sich 9 cylindrische, nach oben etwas verbreiterte Äste von 10-16 mm Höhe. Der längste ist an der Basis 4, am obern Ende $7 \mathrm{~mm}$ breit. Ihre Oberfläche ist schwach längs gerieft. Die Polypen sind so verteilt, daß die kleinern mehr am Rand, die größern mehr in der Mitte der Endscheibe stehen. Ihre Länge beträgt bis $9 \mathrm{~min}$, ihre Breite gegen $2 \mathrm{~mm}$. Die Tentakel $\mathrm{n}$ sind bis $8 \mathrm{~mm}$ lang und $0,285 \mathrm{~mm}$ breit. Ihre Axe ist nicht zungenförmig wie bei der Mehrzahl der Xeniiden, sondern cylindrisch. Die Pinnulae sind dick und stumpf, selır locker angeordnet, stehen in 3 Reihen zu beiden Seiten der Mittellinie und lassen die Axe in ihrer ganzen Länge frei. Kalkkörper habe ich nicht gefunden. Die F arbe der Kolonie ist graugrün, im Leben sind die Tentakeln dunkelgrauviolett.

Fundort: Tumbatu, Südriff. 1 Exemplar.

\section{Litteratur:}

\section{Xenia elisabethae (Köll.).}

1) KöLLIKER, Die Pennatulide Umbellula und zwei neve Typen der Alcyonarien. Festschr. z. Feier des 25 jähr. Best. d. phys. med. Gesellsch. i. Würzburg. Würzburg 1874 , S. 12 .

2) KLUNZINGER, Die Koralltiere d. rot. Meeres. Teil I. Berlin 187\%. S. 41.

3) IIAACKE, Zur P'hysiologie der Anthozoen. Zool. Gart. XX VII Jahrg. Frkft. a. M. 1886. S. 285.

4) STUDER, Versuch eines Systems der Alcyonaria. Arch. f. Naturg. 53. Jahrg. I. Bd. Berlin 188\%. S. 14.

5) WRIGHT u. S'TUDER, Report on the Alcyonaria collected by H. M. S. Challenger. Chall. Report, Zool. Vol. XXXI. 1889. S. XVII.

6) SCHENK, Clavulariiden, Xeniiden u. Alcyoniiden von Ternate. Frkft. a. M. 1896. S.52. Synonyma: Heteroxenia elisabethae KöLL. (1).

Diagnose: Pinnulae in 4 Reihen auf jeder Seite der Mittellinie der Tentakeln, ziemlich kurz und dick. Axe der Tentakeln in ihrer ganzen Länge frei. 
Beschreibung: Von dieser Spezies hat KöLLKer (1) eine so ausgereichnete und eingehende Beschreibung geliefert, dafis ich lier einfach darauf verweisen kann. In dem Material des Hamburger Museums fand ich ein sehr schönes Exemplar, das selbst bis auf die Mafßangaben mit der KüLLLKer'schen Beschreibung übereinstimmt. Nur die von KöLlıker beschriebenen Kalkkörper konnte ich nicht auffinden. Die l'innulae sind bald länger und schlanker, bald kürzer und stumpfer, Unterschiede, die mir nicht genügend erschienen, um besondere Spezies darauf zu hegründen. Die Grenzen zwischen Xenia elisabethae KüLL. und Xenia fuscescens EHrBg. sind kaum festzustellen, im wesentlichen sind es nur die verschiedenen Dimensionen, die eine Unterscheidung beider Arten erlatuben. Doch giebt es hier Übergänge, wie von mir untersuchte Exemplare des Berliner Museums zeigen. Es ist daher bis zu einem gewissen Grade Sache des persönlichen Taktes, ob man beide Arten trennen will oder nicht. In meinem Material fanden sich 2 kleine Exemplare aus Tumbatu, die man als $X$. fuscescens bezeichnen kann.

Fundort: Sansibar, 1 sehr schönes großes Exemplar.

\section{Xenia membranacea Schenk.}

Litteratur:

SCHENK, Clavulaiiiden, Xeniiden und Aleyoniiden von Ternate. Frkft. a. M. 1896, S. 60 .

Diagnose: Pinnulae in 4 Reihen auf jeder Seite der Mittellinie der Tentakeln, lang und schlank. Axe der Tentakeln nur im untern Teile frei.

Beschreibung: Xenia membranacea Schenk stand mir im Originalexemplar zur Verfigung. Trotz eines ziemlich verschiedenen äußern Habitus glaube ich damit 2 Exemplare des Hamburger Museums identifizieren zu dürfen. Die beiden Kolonieen sind in geringer Entfernung von einander auf einem Madreporenstock aufgewachsen. Der cylindrische Stamm der einen ist unverästelt, $17 \mathrm{~mm}$ hoch und $9 \mathrm{~mm}$ breit, der Stamm der andern teilt sich in $8 \mathrm{~mm}$ Höhe in 2 Äste. Die Polypen stehen so dichtgedrängt auf der Endscheibe, daß ihre Basalenden sich berühren. Sie erreichen eine Länge bis zu $7 \mathrm{~mm}$, eine lireite bis zu $1 \mathrm{~mm}$. Am Rande der Scheibe stehen zahlreiche kleine unentwickelte Polypen, diese fehlen in cler Mitte. Die Tentakeln sind bis $6 \mathrm{~mm}$ lang und haben eine zungenförmige Axe. Die langen Pinnulae stehen in 4 Reihen jederseits der Mittellinie der Tentakeln und lassen nur den untern Teil der Axe frei. Die untersten I'innulae haben Wärzchenform. Die Kalkkörper meiner Exemplare sind sehr zahlreich, während sie bei dem Schexk'schen Exemplar nur ganz vereinzelt auftreten. Die F arbe der Kolonieen ist hellgell.

Fundort: Sansibar, 1 Exemplar. 


\section{Xenia quinqueserta n. sp.}

Diagnose: Pimnlae in 5 Reihen auf jeder Seite der Mittellinie der 'Tentakeln, selır kurz, dick und stumpf. Axe der 'Tentakeln zungenförmig, in ihrer ganzen Länge frei.

Beschreibung: Auf einem Bruchstïck einer Muschelschale erheben sich in geringen Abständen \ Kolonieen. Der cylindrische glatte Stamm ist bei einer der Kolonicen $7 \mathrm{~mm}$ hoch und $15 \mathrm{~mm}$ breit, bei einer anderu $13 \mathrm{~mm}$ hoch und $10 \mathrm{~mm}$ dick. Die I'olypen stehen dichtgedrängt auf der obern Fläche des Stammes und sind ohne die Tentakeln bis $13 \mathrm{~mm}$ lang und $2,5 \mathrm{~mm}$ breit. Es finden sich alle Übergänge in der Größe von kleinen Knospen bis zu der genannten Länge. Der Polypenkörper ist neist quergerunzelt und am obern Ende etwas verbreitert. Die Tentakeln sind bis $8 \mathrm{~mm}$ lang und an der Basis $1 \mathrm{~mm}$ breit. Die Pinnulae stehen in 5 Reihen auf jeder Seite der Mittellinie. Sie sind kurz, dick und stumpf und lassen die Tentakelaxe in ihrer ganzen Länge frei. Kalkkörjer fehlen. Die Farbe der Kolonie ist hellgrau.

Fundort: Tumbatu. Eine Muschelschale mit mehreren Kolonicen.

\section{Xenia baniana n. sp.}

Diagnose: Pinnulae auf beiden Flächen der 'Tentakeln angeordnet, in 3 Reihen auf jeder Seite der beiden Mittellinien, lang, schlank und spitz. Axe der Tentakeln auf beiden Seiten in ihrer ganzen Länge frei.

Beschreibung: Es liegt mir nur ein Exemplar vor, dessen dicker fleischiger unvollständig erhaltener Strunk oben scheibenförmig ausgelbreitet ist und hier $47 \mathrm{~mm}$ Länge und $33 \mathrm{~mm}$ Breite erreicht. Die Polypen stehen dichtgedrängt auf der Endscheibe und sind ohne die Tentakeln bis $20 \mathrm{~mm}$ lang und $2 \mathrm{~mm}$ breit. Die zungenförmigen T'entakeln werden bis $10 \mathrm{~mm}$ lang. Die langen und schlanken Pinnulae sind zu beiden Seiten von zwei Mittellinien angeordnet, einer äußern und einer innern. Sie erstrecken sich daher gleichmäßjig auf die äußere und innere Flïche der Tentakeln und lassen auf beiden Seiten nur einen schmalen Teil der Axe frei. Ich zählte etwa 6 Reihen Pinnulae auf jeder Seite der Tentakelaxe. Kleine knospenförmige Polypen fand ich fast nur am Scheibenrand. Die Kalkkörper haben die gewöhnliche Form und Größe. Sie sind besonders in den Tentakelı sehr dicht gehäuft. I)ie Farbe der Kolonie ist hellbraun.

Fundort: Insel Baui. 2 Exemplare.

\section{Xenia medusoides n. sp.}

Diagnose: Pinnulae rings um die schlanke Tentakelaxe angeordnet, ohne erkennbare Mittellinie, sehr lang, schlank und spitz.

Beschreibung: Kin $14 \mathrm{~mm}$ hoher, $8 \mathrm{~mm}$ breiter cylindrischer Strunk erhebt sich an der einen Scitenlinie einer rhombischen Membran von $12 \mathrm{~mm}$ Länge, die auf einem Madreporenast befestigt ist. Auf demselben 
Ast steht noch eine etwa grleich grofse und eine kleinere Koolonie. Auf einem zweiten Ast sind awei gröfere aber schlecht erhaltene lixemplare aufgewachsen. Die mit 8 weilsen Längslinien versehenen P'olypen sind bis $15 \mathrm{~mm}$ lang und $2 \mathrm{~mm}$ breit. Kwischen ihnen finden sich zahlureiche junge Knospen. Die Tentakeln erreichen eine Jänge bis zи $9 \mathrm{~mm}$. I) sehr langen, schlanken und spitzen I'innulae sind rings un die schlanke Tentakelaxe angeordnet. I) Kalkkörper haben die bekannte: Form und sind gleichmäßig in der ganzen Kolonie verteilt. I) Farbe der Kolonie ist grau.

Fund ort: Tumbatu, Südriff; 2 Exemplare.

I.itteratur:

\section{Gattung: Cespitularia Val.}

1) QUOY et GAIMARD, Voyage de l'Astrolahe, Zool. T. IV, Paris 1833, S. 265. PI. 22, Fig. 1-7.

2) MILNE-EDWARDS, Hist. nat. d. Cor. T. I, Paris 1857, S. 126.

3) BRUNDIN, Aleyonarien a. d. Sammlung d. zool. Mus. in Upsala, Bihang till Svenska Vet. Akad. Handl. Bd. 22, Afd. IV, Nr. 3. Stockholm 1896, S. 4.

Synonyma: Cornularia Q. G. (1).

\section{Suensonia BRUndin (3).}

Diagnose: Xeniiden von baumförmigem Habitus.

Historisches: Die erste Art dieser Gattung wurde 1833 von (Quoy und Gamard (1) als Comularia mullipinnata beschrieben. Auf sie gründet später Valfactereses für ein Exemplar des Pariser Museums dats neue Genus, das aher von keinem der spätern Autoren anerkannt wirl. Ich stelle es hier wieder her und vereinige mit ihm die 189)(i von Brrixnis (3) geschaffene Gattung Suensoniu. Das Genus umfalit bis jetat 5 Arten, darunter die folgende neue meines Materials.

\section{Cespitularia coernlea n. sp.}

Diagnose: Pinnulae in (iner Reihe anf jeder Seite der Mittellinie der Tentakeln. Polypen (olne T'entakeln) $4 \mathrm{~mm}$ lang, Tentakeln 2,5 mm lang. Kalkköıper fehlen.

beschreibung: Fin steriler Strunk von $50 \mathrm{~mm}$ Höhe und $35 \mathrm{~mm}$ mittlerer Breite spaltet sich am ohern Ende in 4 gerade in die Höhe gehende Ïste, deren jeder sich wieder in 2 ebenfalls aufwärts strebende, $15-45 \mathrm{~mm}$ lange Äste teilt, an denen die l'olypen :ïhrenförmig angeordnet sitzen. Die Polypenkärper sind $4 \mathrm{~mm}$ lang. $1 \mathrm{~mm}$ breit, die Tentakeln $2,5 \mathrm{~mm}$ lang, die P'innulate his $0,6 \mathrm{~mm}$ lang und in einer Reihe auf jedter Seite der Tentakelmittellinie angeordnet. Kalkkörper fehlen. Als Farhe der lebenden Kolonic ist angegehen: Stamm und Polypen fleischfarben, alle hellblau angehaucht. besonder's die Polypenröhren. Tentakeln nicht dunkler.

Fundort: Kokotoni: 1 Exemplar. 
Litteratur:

\section{Fam.: Alcyoniidae Verrill.}

1) LINNÉ, Systema naturae. Tom. I, Editío X. Holmiae 1758. S. 803.

2) PALLAS, Elench. zooph. 1766, S. 242.

3) LAMARCK, Hist. Nat. des animaux sans vert. Tome II. Paris 1816. S. 388 u. 412.

4) LAMOUROUX, Expos. méth. des genres de l'ordre des Polypiers. Paris 1821, S. 68 ff.

5) LESSON, Voyage de la Coquille. Zool. Tome II, Zooph. 1831, S. 92.

6) LESSON, Voyage aux Indes orientales. Zool. Zooph. 1834, S. 517.

7) EHRENBERG, Die Korallentiere des roten Meeres, Berlin 1834, S. 56.

8) GRAY, Proc. Zool. Soc. Lond. 1835, S. 60.

9) MILNE-EDWARDS, Ann. des Sci. Nat. ser. 2, Tome IV, 1835, S. 323.

10) DANA, Zoophytes, Philadelphia 1846, S. 611.

11) MILNE-EDWARDS, Hist. Nat. des Corr. Tome I, Paris 185\%, S. $113 \mathrm{ff}$.

12) DANA, Synopsis, New Haven 1859, S. 119.

13) GRAY, Proc. Zool. Soc. London 1862, S. 35.

14) VERRILL, Proc. Essex Inst. Vol. IV, No. V, 1865, S. 148.

15) KÖLLIKER, Verhandl. der Phys. Med. Gesellsch. Würzburg $186 \%$.

16) VERRILL, Proc. Essex Inst. Vol. VI, 1869, S. 46.

17) GRAY, Notes on the fleshy Alcyonoid Corals. Ann. and Magaz. of Nat. Hist. Vol. III. 4. ser, 1869 , S. $121 \mathrm{ff}$.

18) KENT, Quart. Journ. Micr. Sci. VoI. XVIII, 1876, S. 397.

19) KLUNZINGER, Die Koralitiere des roten Meeres, I. Bd. Berlin 1877, S. 21.

20) VERRILL, Amer. Journ. Sci. and Arts. Vol. XVI, 1878. S. 376.

21) STUDER, Alcyonarien der Gazelle. Monatsber. d. k. preuss. Akad. d. Wiss. Berlin 1878, S. 633.

22) W. KOCH, Neve Anthozoen, Marburg 1886, S. 3.

23) MARENZELLER, Über die Sarcophytum benannten Alcyoniiden. Zool. Jahrb. I. Bd. 1886. S. 341.

24) DANIELSSEN, Norske Nordhavs-Exped. 1876-78. Zool. Alcyonida 1887. S. 74, 118 u. 129.

25) S'TUDER, Versuch eines Systems der Alcyonarien. Arch. f. Naturg. 53. Jahrg. I. Band, 188\%. S. 14 .

26) WRIGHT and STUDER, Report on the Alcyonaria collected by H. M. S. Challenger. Chall. Report, Zool. Vol. XXXI, 1889, S. XVIII u. 238.

27) PFEFFER, Zur Fauna von Süd-Georgien. Jahrb. d. Hamb. Wiss. Anst. VI. Jahrg. 2. Hälfte. 1889. S. 49 .

28) KOCH, Die Alcyonaceen des Golfes v. Neapel. Mitt. aus d. zool. Stat. zu Neapel. 9. Bd., 4. Heft, 1890.

29) BRUNDIN, Alcyonarien aus der Sammlg. des Zool. Mus. in Upsala. Bihang till Svenska Vet. Akad. Handlingar. Bd. 22. Afd. 1V, No. 3. Stockholm 1896.

Synonyma: Polypi tubiferi LM. (pars) (3).

Alcyonées LaM. (pars) (4).

Halcyonina EнRвg. (pars) ( 7 ).

Alcyoninae DANA (pars) (10 u. 12).

Alcyoninae M.-E. (pars) (11).

Alcyonidae Verrill (pars) (14).

Lobulariadae + Alcyoniadae + Sarcophytidae + Bellonelladae Gr. (16). 
Alcyoninae retractiles KLZGR. (19).

Alcyonidae TH. STUd. (25).

Alcyonidae Stud. Wreht. (26).

Diagnose: Alcyonaceen mit reichlich entwickeltem Coenenchym, das die P'olypen meist bis zu dem retraktilen Oesophagealteil umgiebt. Basalteil der Kolonie ohne P'olypenöffnung. Polypen durch verästelte Ernährungskanäle verbunden, die in verschiedener Höhe über der Basis der Kolonic verlaufen. Dic jüngern Polypen entspringen den weiter von der Basis entfernten Kanälen. Polypen daher von sehr ungleicher Länge. Bei mehreren Gattungen Dimorphismus. Spicula sehr verschieden.

Systematik: Ich habe in der Litteratur 58 Alcyoniidenarten aufgefunden, zu denen noch 5 hier beschriebene neue kommen, sodaß sich die Gesamtzahl der Arten auf 63 beläuft. Viele der älteren Spezies sind jedoch so ungenügend charakterisiert, daß es zweifelhaft ist, ob sie alle gut begründet sind. Die 63 Arten verteilen sich auf 11 Gattungen, deren charakteristische. Merkmale sich aus folgender Tabelle ergeben.

I. Kolonieen ohne Dimorphismus der Polypen:

A. Der obere Teil der Kolonie ist nicht in den untern zurückziehırar.

1. Kolonie cylindrisch, mit halbkugligem Kopf, der mit Polypen bedeckt ist:

Nidalia Gr.

2. Kolonie blumenkohlartig, mit aufrechtem Strunk und flacher vielfach gefalteter Scheibe, auf deren Oberfläche die vollständig retraktilen Polypen stehen:

Sinularia n. g.

3. Kolonie massig, im obern Teil lappig geteilt:

Alcyonium L.

4. Kolonie mit aufrechtem Stamm, dessen oberes Ende mit zahlreichen Polypen besetzt ist. Kurze Äste in Wirteln um den Stamm geordnet, mit Polypen an den verdickten Enden. Kelche durch breite Coenenchymstreifen getrennt: Krystallofancs DAN.

5. Habitus wie bei 4, aber die Kelche durch wenig Coenenchym getrennt:

Sarakka DAN.

6. Kolonie baumförmig verästelt. Polypen auf der ganzen Oberfläche verteilt.

Daniela v. Koc日.

๘. Kolonie keulenförmig, mit hervorragenden Warzen des Coenenchyms bedeckt, aus denen die Polypenkëpfe rorragen.

Metalcyonium PeEfFer. 
B. Der obere Teil der Kolonie ist in den untern zurückziehbar: Paralcyonium M.-E.

II. Kolonieen mit Dimorphismus der Polypen:

A. Autozooide zahlreich.

1. Scheibe der Kolonie hutpilzförmig, gefaltet. Spicula mit unregelmäßig angeordneten Warzen.

Sarcophytum LEsS.

2. Obere Fläche der Kolonie zu Lappen, Läppchen und fingerförmigen Fortsätzen ausgewachsen. Spicula mit gürtelförmig angeordneten Warzen:

Lobophytum Marenz.

B. Autozooide spärlich:

Anthomastus VerRiLl.

\section{Gattung: Sinularia nov.}

Diagnose: Steriler Strunk aufrecht, im obern Teil mit sehr tiefen Längsspalten versehen. Scheibe vielfach gefaltet. Spicula des Innern reich mit Warzen besetzte Spindeln von sehr verschiedener Länge. Spicula der Rinde keulenförmig, mit stark entwickeltem und reich differenziertem Kopf.

Diagnose der Gattung.

\section{Sinnlaria brassica n. sp.}

Beschreibung: Die Kolonie besteht aus einem aufrechten sterilen Strunk und einer beinahe horizontalen die Polypenöffnungen tragenden Scheibe. Die größte Höhe des Strunks beträgt $50 \mathrm{~mm}$, die kleinste $30 \mathrm{~mm}$. An der Basis ist der Strunk nur $19 \mathrm{~mm}$ breit, am Scheibenende dagegẹn über $40 \mathrm{~mm}$. Seine Oberfläche ist ziemlich glatt und nur mit schwachen Querrunzeln bedeckt. Am obern Teil des Strunkes sind mehr oder weniger weit herabgehende tief eingeschnittene Längsspalten vorhanden, die den Anschein erwecken, als ob der Strunk aus mehreren Stämmen zusammengewachsen wäre. Die Scheibe ist im allgemeinen von elliptischer Form, flach und bis in die Mitte vielfach gefaltet. Die Falten werden durch die tiefen Längsspalten, die sich auf den Strunk fortsetzen, bedingt. Sie gehen von allen Seiten des Randes aus und stoßen in der Mitte zusammen. Die Scheibe ist $53 \mathrm{~mm}$ lang, bis $32 \mathrm{~mm}$ breit und $2 \mathrm{~mm}$ hoch. Die Polypen sind sämtlich vollständig eingezogen, und man sieht auf der Oberfläche der Scheibe nur die in unregelmäßigen Reihen angeordneten Mündungen. Die Spicula der Kolonie sind von zweierlei Art. Im Innern finden sich bis $4 \mathrm{~mm}$ lange und bis $0,855 \mathrm{~mm}$ breite Spindeln, die sehr reich mit bedornten Warzen besetzt sind. Die Größse ist auferordentlich verschieden. Neben diesen Spindeln kommen mehr stabförmige Spicula vor, die spärlicher mit weniger differenzierten Warzen besetzt sind. Die Spicula der Rinde 
sind 0,18 mm lange, 0,04 $\mathrm{mm}$ breite heulen, deren bis 0,1 mm lneriter Kopf sehr stark differenziert ist. 1)ie l'arbe der Kolonie ist hollogolblich. Fundort: Tumbatu, ein gut erhaltenes Exemplar.

Litteratur:

\section{Gattung: Alcyonium L.}

1) LINNÉ, Systema naturae, Tom. I. Editio X, Holmia 1758. S. 803.

2) PALLAS, Elench. zooph. 1766. S. 242.

3) LSI'ER, Die P'llanzentiere. Nürnberg, 1791-1797. III. Teil, S. 3. Taf. I his XXV.

4) LAMARCK, Hist. Nat. des animaux sans vert. Tome II. Paris 1816. S. 388.

5) IAMOUROL:X, lixpos. míthodique des genres de l'ordre des L'olypiers, l'aris 1821, S.70fr.

6) QUOY et GAIMARD, Voyage de l'Astrolabe. Zool. 'Tome IV, Paris 1833, S. 269.

7) EHRkNBERG, Die Korallentiere des roten Meeres, Berlin 1834, S. $5 \%$

8) DANA, Zoophytes, Philadelphia 1846, S. 611.

9) MILNE-EDWARDS, Hist. nat. des Coralliaires, Tome I, Paris 185\%, S. 114.

10) DANA, Synopsis, New Haven 1859, S. 122.

11) GRAY, Notes on the fleshy Alcyonoid Corals. Ann. and Mag. of Nat. Hist. Vol. III. 4. ser. 1869 , S. $121 \mathrm{ff}$.

12) KLUNZINGER, Die Koralltiere des roten Meeres, I. Bd., Berlin 187\%, S. 21.

13) WRIGHT and STCDER, Report on the Alcyonaria collected ly H. M. S. Challenger. Chall. Report, Zoologie, Vol. XXXI, 1889. S. XX u. 238.

Synonyma: Lobularia Sav. (apud Lamarck (4).

Chlorozoa + Amicella + Alcyonium + Danella + Amocella + Cladiella Gr. (11). Alcyonium + Lobularia STUD. Whrgt. (13).

Diagnose: Die Kolonicen bilden flach ausgebreitete oder aufrechte Massen, die auf der lappig geteilten Oberfläche die vollständig retraktilen Polypen tragen. Kein Dimorphismus der Personen.

\section{Aleyonium polydactylum (Ehrbg.).}

\section{Litteratur:}

1) EHRENBERG, Die Korallentiere des roten Meeres. Berlin 1834, S. 59.

2) DANA, Zoophytes, Philadelphia 1846, S. 617.

3) MILNE-EDWARDS, Hist. nat. des Corall. Tome I, Paris 185\%, S. 121.

4) DANA, Synopsis, New-Haven 1859, S. 124.

5) GRAY, Notes on the Fleshy Aleyunoid ('orals. Annals and Manazine of Yat. Ilist. Vol. III, 4. Ser. 1869.

6) KLUNZINGER, Die Koralltiere des roten Meeres, Berlin 1877, S. 26.

7) S(HFNK, Clavulariden, Xeniiden uni Aleyoniiden von T'mate. Frkft. a. M. 18946. S.69.

Synonyma: Lobularia polydactyla Eнrвg. (1).

Alcyonium polydactylum DANA (2 u. 4).

Amocella polydactyla GR. (5).

Diagnose: ('oenenchym grobkërnig. Iaijpehen kïr\%er oder länger fingerfürmig oder fast eiförmig, gedrängt, zu Ialppen gruppiert. die sich aus dem oft sohr umfangreichen Basalteil hügrelartig erheben. Kalkkërper des Innern his $2 \mathrm{~mm}$ lange. mit zahlreichen bedornten Warzen besetzte Spindeln. Kalkkörper der Rinde keulenfürmig. mit locker stehenden Dornen. 
Historisches: Enrenbera. (1) beschreibt zuerst diese Art ihrer äußern Form nach, KLunzinger (6) giebt die Beschreibung und Abbildung der Spicula. GRAY (5) stellt sie mit Alcyonium pauciflom und A. trichanthinum zu seiner Gattung Amocella.

Fundort: Sansibar, 3 Exemplare. Insel Baui: 1 Exemplar.

\section{Litteratur:}

\section{Gattung: Sarcophytum Less.}

1) LINNÉ, Systema naturae. Tom. I, 1758, S. 803.

2) SAVIGNY, Iescription de l'Egypte. Hist. nat. Tome I, 4, partie. Paris. S. 22\%. Die übrige Litteratur bis 1886 siehe bei MARENZELLER.

3) MARENZFLTLR, Über die Sarcophytum benannten Alcyoniiden. Zool. Jahrb. I. Bd. Jena 1886. S. 341.

4) WRIGHT and STUDER, Report on the Alcyonaria collected by H. M. S. Challenger. Challenger-Report, Zool. Vol. XXXI, S.XXI u. $244 \mathrm{ff}$.

5) SCHENK, Clavulariiden, Xeniiden u. Alcyoniiden von Ternate. Frkft. a. M. 1896, S. $70 \mathrm{ff}$.

Synonyma: Alcyonium L. (pars) (1).

Lobularia Sav. (pars) (2).

Sarcophyton LESS. (3).

Halcyonium EнRвg, (3).

Diagnose: Siehe Marenzeller (3).

\section{Litteratur:}

\section{Sarcophytum glanenm (Q. G.).}

1) QUOY et GAIMARD, Voyage de l'Astrolabe. Paris 1833. T. IV, S. 270, Zoophytes Pl. XXII, Fig. 11, 12.

2) DANA, Zoophytes. 1846, S. 623, pl. 58, Fig. 4 u. pl. 59, Fig. 6.

3) MILNE-EDWARDS, Hist. nat. des Coralliaires, Tome I, Paris 185\%, S. 121.

4) DANA, Synopsis, New Haven 1859. S. 125.

5) GRAY, Notes on the fleshy alcyonoid corals. Ann, and Magazine of Natural Hist. Vol. III. 4. ser. Lond. 1869, S. 125 .

6) STUDER, Alcyonarien der Gazelle. Monatsbericht der Akad. d. Wiss. zu Berlin. Okt. 1878. S. 634 .

7) MARENZELLER, Üher die Sarcophytum benannten Aleyoniiden. Zool. Jahrb. I. Band. Jena 1886. S 3วั2.

8) WRIGHT u. STUDER, Report on the Alcyonaria collected by II. M. S. Challenger, Chall.-Report. Zool. Vol. XXXI, S. 248.

Synonyma: Alcyonium glaucum Q. G. (1).

Diagnose: Strunkspicula spindelförmig mit zahlreichen bedornten Warzen. Scheibenspicula stabförmig, mit einfachen Warzen.

Historisches: Diese Art wurde zuerst 1833 von Qoox und Galmard (1) als Alcyonium glaucum beschrieben. Gray (5) stellt sie 1869 als Sarcopliyton glaucum zu seiner Familie der Sarcophytiden. Die erste ausführliche Beschreibung giebt 1886 Marenzeller (7). Ich stelle 5 Exemplare meines Materials zu dieser Art. Sie stimmen in den wichtigsten Spiculaformen überein, sind aber im Habitus sehr verschieden. Ich gebe daher eine kurze Charakteristik der einzelnen Formen: 
1) Ein Exemplar aus Kokotoni: Stiel sehr unregelmälisig gestaltet, etwa $40 \mathrm{~mm}$ hoch, Umfang etwa $200 \mathrm{~mm}$. Lïnschluf zahlieicher Fremclkörper. Seheibe annähernd kreisförmig, Durchmesser etwa $100 \mathrm{~mm}$, Dicke etwa $5 \mathrm{~mm}$. Zahlreiche bis in die Mitte der Scheibe gehende Falten. Der Rand der Scheibe ragt bis gegen $40 \mathrm{~mm}$ vor. Etwa 6 Autozooide auf $1 \mathrm{~cm}$ Lïnge, Mehrzahl ganz eingezogen, ein Teil bis $5 \mathrm{~mm}$ ausgestreckt. Miundungen der Siphonozooide deutlich mit blofem Auge sichtbar. Furbe braun.

Ein zweites Fxemplar von demselben Fundort hat denselhen Habitus, aber bedeutend geringere Dimensionen.

2) Ein sehr groß3es Fxemplar aus T'umbatu. Stiel $90 \mathrm{~mm}$ hoch, $70 \mathrm{~mm}$ breit, sehr fest. Scheibe bis fast in die Mitte gelappt, $130 \mathrm{~mm}$ lang, $100 \mathrm{~mm}$ breit, $6 \mathrm{~mm}$ dick. Ihre beiden Hälften sind gegen einander in die Höhe gehoben und berühren sich mit den obern Flächen. 7 Autozooide auf $1 \mathrm{~cm}$ Länge, die meisten ausgestreckt, $6 \mathrm{~mm}$ lang. Spicula der Polypen gleich denen der Scheibe. Die spindelförmigen Spicula des Strunkes sind Mittelformen zwischen denen von S. glancum und paupereulum. Farbe braun.

Damit stimmt im wesentlichen ein kleineres Exemplar desselben Fundorts überein.

3) Ein Exemplar von der Insel Changu bei Sansibar: Scheilee steht nicht uiher den Stiel üher. Stiel $100 \mathrm{~mm}$ lang, $60 \mathrm{~mm}$ breit, $40 \mathrm{~mm}$ hoch. Scheibe am Rand sehr stark gefiltet, dir nach imnen rorspringenden Falten wölben sich über dem umgefalteten Teil der Scheibe zusammen und bedecken ihn. Gegen 10 Autozooide auf $1 \mathrm{~cm}$ Lü̈nge, alle eingezogen. Siphonozooide sehr klein, nur an wenigen Stellen mit bloß3em Auge sichthar. Farle grau.

\section{Sarcophytum trocheliophorum Marenz.}

\section{Litteratur:}

var. amboinense Marenz.

1) MARKAZELLER, Über die Sarcophytum benannten Alcyoniiden. Zool. Jahrlb. I. Bd. 1886 , S. 361.

2) WRIGHT and STEDER, Report on the Alcyonaria collected by II. M. S. Challenger, Chall. Report, Vol. XXXI, 1889, S. 249.

Diagnose: Strunksjicula dichtwarzige Walzen, ohne mittlere Einschnürung, grösser als die der Stammform (Marknzelder, Tuf. IX, Fïg. 6 c.).

Beschreibung: Auf Grund der Natelformen, namentlich der charakteristisch gestalteten Spicula des Strunkes, stelle ich zu dieser Varietät 5 Exemplare, die in ihrem Habitus sehr verschieden sind. Besonders sehen die Mündungen der Autozooide sehr verschieden aus, doch scheint dies an dem verschiedenen Kontraktionszustand zu liegen, da man die gleichen Unterschiede auch bei den verschiedenen I'olypenmündungen derselben Kolonie antrifft. Ich habe daher kein besonderes ('iewicht darauf gelegt.

1) Ein Exemplar aus Kokotoni: Typische Hutpilzform. Stiel 36 bis $66 \mathrm{~mm}$ hoch, im untern Teil $40 \mathrm{~mm}$ breit, mit unregelmäßigen schwachen 
Längsriefen. Scheibe sitzt sehr stark geneigt auf dem Stiel, ist annähernd kreisförmig, ragt bis $35 \mathrm{~mm}$ über den Stiel vor. Durchmesser $70 \mathrm{~mm}$, Dicke $5 \mathrm{~mm}$. Rand der Scheibe nach abwärts gekrümmt, in 8 teils größere, teils kleinere Falten gelegt. Gewebe des Stiels sehr hart, das der Scheibe weicher. Autozooide am Rande der Scheibe in deutlichen Reihen, mehr oder weniger weit eingezogen, etwa 8 auf $1 \mathrm{~cm}$ Länge. Mündungen der Autozooide etwa $1 \mathrm{~mm}$ grof, langgestreckt. Siphonozooidmündungen deutlich mit bloßem Auge sichtbar. Farbe graugrünlich.

Zwei kleinere Exemplare von demselben Fundort haben den gleichen Habitus. Das kleinste hat erst eine Falte.

2) Fin Exemplar aus Sansibar: Sehr stark in die Breite und weniger in die Höhe entwickelt. Stiel $30 \mathrm{~mm}$ hoch, Scheibe selır stark gefaltet. Autozooide vollständig eingezogen, in regelmäßigen Reihen stehend, 10 auf $1 \mathrm{~cm}$ Länge. Mündungen der Autozooide etwa $1 \mathrm{~mm}$ lang, sehr schmal. Mündungen der Siphonozooide deutlich mitblofem A uge sichtbar. Farbe gelblich.

3) Ein Exemplar ohne nähere Fundortsangabe (Ostafrika): Stiel sehr stark verbreitert, auf Madreporenästen aufgewachsen. Scheibe annähernd kreisförmig, Durchmesser $100 \mathrm{~mm}$, am Rande stark gefaltet. Autozooide vollständig eingezogen, auf dem mittlern Teil der Scheibe ziemlich unregelmäßig und in größern Fntfernungen von einander stehend, am Rand dichter und in Reihen angeordnet, etwa 8 auf $1 \mathrm{~cm}$ Lïnge. Öffnungen der Autozooide in der Mitte der Scheibe $2 \mathrm{~mm}$ lang, $1 \mathrm{~mm}$ breit, von elliptischer Form, am Rand kleiner. Siphonozooidmündungen sehr deutlich sichthar.

Litteratur:

\section{Gattung: Lobophytum Marenz.}

1) MARENZELLER, Über die Sarcophytum benannten Aleyoniiden. Zool. Jahrb. I. Bd. $1886, \mathrm{~S} .341$.

2) WRIGIT and STUDFR, Report on the Aleyonaria collected by II. M. S. Challenger. Chall. Rep. Vol. XXXI, 1889, S. XXI u. 250.

Synonyma: Alcyonium L. Sarcophytum LEss.

Diagnose: Siehe Marenzeluer (1).

\section{Lobophytum crassum Marenz.}

var. sansibaricum nov.

Diagnose: Die Rindenspicula sind Keulen mit zahlreichen Warzen, viel komplizierter als die der Stammform. Strunk- und Scheibenspicula gleich denen der Stammform.

Beschreibung: Mir liegen ror: 4 Exemplare von verschiedener Größse aus Sansibar. Beim größten ist der Strunk $45 \mathrm{~mm}$ hoch, der Durchmesser der kreisförmigen Scheibe $140 \mathrm{~mm}$ lang. Die Lappen crstrecken sich bis in die Mitte der Scheibe und sind in zahlreiche fingerförmige 
Fortsiitze unsgewuren. A utozonide sämtlich vollständig zurtickgezogen, in melır oder weniger dentlichen Reihen stehend, gegen 8 auf $1 \mathrm{~cm}$ Länge. Mündungen der Siphonozonide mit blofem Auge deutlich sichthar. Die Rindenspieula sind Kenlen mit deutlichen Wargengürteln, 0,14 mm lang und $0,0.28$ mm breit. I)ie andern sipicula stimmen im wesentlichen mit den von Marenzaliter für Lobopliytum crassum abgebildeten üherein.

1 Exemplar ans T'umbatu reigt keine bedentenden Abweichungen.

\section{Fannilie: Nephthyidae Verrill.}

\section{Iit teritur:}

Die Litteratur bis 1896 siche bei KüKLNTHAI, (1) unl MAY (3).

1) kïklithis, Aleyonaceen von 'Ternate. lirkt. a. M. 1896.

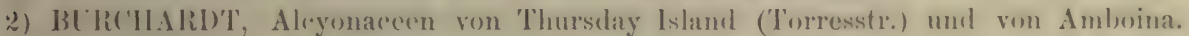
In : Sl:Dlox, Zoul. Forschungseisen in Australien u. d. Matayisehen Arehipel, V. Bel., IV. Liefereg. Jena 1898 , S. 431.

3) MAY, Aleyonaceen von Ost-Spitzbergen. Zool. Jahub. Bd. XI, Jena 18!98, S. 385.

Synonyma: Spoggodinae + Ammothea + Vephthya, I)ana $(1,12)$.

Alcyoniens armés + Ammothea, M.-E. $(1,11)$.

Spoggodidae + Nephthyadae + Lemnaliadae (pars), GrAY $(1,19)$.

Nephthyidae VERriLL $(1,18)$.

Alcyoninae capituliferae KLZGR. $(1,21)$.

Nephthyidae + Siphonogorgiaceae, Tн. STud. (1,27) und STUD. WRGhT. $(1,28)$.

Diagnose: siehe Kükentrat (1).

Systematik: Die Familie der Nephthyiden umfifist nach musern jetzigen Kenntnissen 143 Arten. Diese verteilen sich auf 4 Gattungen:

I. Polypen ohne Stützbündel.

A. Polypen in Kätzchen: Ammothea Sav.

B. Polypen in Bündeln oder einzeln: Paraspongodes Коктн.

II. Polypen mit Stützbündel:

A. Polypen in Kätzchen : Nephithya Sav.

B. Polypen in Bündeln oder einzeln: Spongodes Less.

I.itteratur:

\section{Gattung: Ammothea Sav.*}

1) Foliskita, I)eseriptiones animalium. Hauniae 17\%5, s. 1339.

2) LAMAlick, Histuire naturelle des animatux sans rert. T. II, P'aris 1816, s. flo.

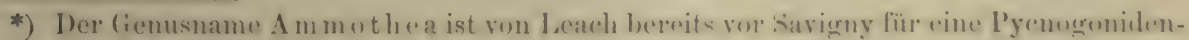
grattung eingeführt worden. Ich habe aber nicht gewagt, den einmal eingebürgerten Camen hier zu ändern. 
3) SAVIGNY, Déscr. de l'kigypte. Hist. Nat. Paris; Pl. T. II, Polypes, Taf. 2, Fïg. 6, Paris $181 \%$.

4) LAMOUROUX, Exp. méth. des genres de l'ordre des Polypiers, Paris 1821, S. 69.

5) BLAiNVILLE, Manuel d'Actinologie, Paris 1834, S. 522.

6) QUOY et GAIMAliL, Voyage de l'Astrolabe, Zool. Paris 1833, S. 275 u. 276.

7) EHRENBERG, Die Korallentiere des roten Mecres. Berlin 1834, S. 59 u. 60.

8) DANA, Zoophytes, Philadelphia 1846, S. $60 \%$.

9) MILNE-EDWARDS, Hist. Nat. d. Cor. T. I, Paris 1857, S. 123.

10) DANA, Synopsis, New-Haven 1859, S. 121.

11) DUChASSAING et MICHELLO'T'T, Mém. sur les Cor. des Antilles, Torino 1860, S. 291.

12) VERRILL, List of the Polyps and Corals sent by the Mus. of Comp. Zool., Harvard College. Cambridge, Vol. I, 1863-69, S. 39.

13) KÖLLIKER, Icones histologicae. II. Abt. 1. Heft, Leipzig 1865, S. 132.

14) GRAY, Notes on the fleshy Aleyonoid Corals. Ann. and Mag. of Nat. Hist. 4. ser. vol. III, 1869, S. 129.

15) HAECKEL, Arabische Korallen, Berlin 1876, S. 44, Taf. I, Fig. 9.

16) MARENZELLER, Die Coelenteraten, Echinodermen und Würmer der k. k. österr.ung. Nordpolexp. Wien 1877, S. 16-22.

17) KLUNZINGER, Die Koralltiere des roten Meeres, Teil I, Berlin 187\%, S. 30.

18) STUDER, Alcyonaceen der Gazelle. Monatsber. d. Kgl. Akad. d. Wiss. i. Berlin, 1878, S. 634.

19) KÜKENTHAL, Alcyonaceen v. Ternate. Zool. Anz. No. 488 u. 489, 1895.

20) KÜKENTHAL, Alcyonaceen von Ternate. Frkft. a. M. 1896, S. 126.

Diagnose: Polypen ohne Stützbündel, in Kätzchen angeordnet.

Zahl der Arten: Man kennt bis jetzt 19 Ammotheaarten, deren charakteristische Eigentümlichkeiten ich in meiner größern Alcyonaceenarbeit übersichtlich zusammengestellt habe.

\section{Ammothea thyrsoides Ehrbg.}

\section{Litteratur:}

1) LAMARCK, Hist. nat. des anim. s. vert. T. II, Paris 1816, S. 412.

2) EHRENBERG, Die Korallentiere des roten Meeres. Berlin 1834, S. 59.

3) I)ANA, Zoophytes. Philadelphia 1846, S. 608.

4) MILNE-EDWARDS, Hist. nat. d. Cor. T. I, Paris 185\%, S. 124.

5) DANA, Synopsis. New-Haven 1859, S. 121.

6) GRAY, Notes on the fleshy Alcyonoid Corals. Ann. and Mag. of Nat. Hist. 4. ser. vol. III, 1869, S. 131.

7) KLUNZINGER, Die Koralltiere des roten Meeres. Berlin 187\%, S. 31.

Synonyma: Ammothea phalloides Ly. (1). Verilliana thyrsoides GR. (6).

Diagnose: Zahlreiche aufrechte cylindrische Stämme auf gemeinsamer Basis. Polypen nicht dichtgedrängt an den fingerförmigen Enden der Stämme, $1-2 \mathrm{~mm}$ lang, $1 \mathrm{~mm}$ breit. Spicula sämtlich schlanke Spindeln mit wenigen sehr kleinen Dornen. Polypenspicula 0,08-0,16 mm lang, $0,016 \mathrm{~mm}$ breit. Rindenspicula $0,38-0,475 \mathrm{~mm}$ lang, $0,02 \mathrm{~mm}$ breit. Spicula der Kanalwände $0,38-0,475 \mathrm{~mm}$ lang, $0,02 \mathrm{~mm}$ breit. 


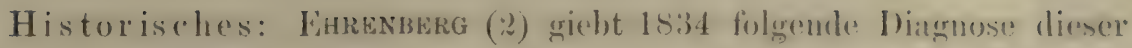
Art: .Batsi carnosil, effusil, supral simpliciter carnosa, ramis cylimblicis, pollicaribus, erectis, verrucosis (omentiformibus)". Line genane Beschreihung

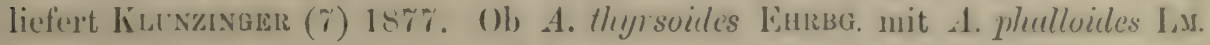
identisch ist, erscheint mir fruglich.

Mir liegen 4 Exemplare aus Tumbatu vor.

Farbenangabe: Stamm grauviolett, Köpfchen heller. Tiefe: 2-3 Faden.

Ferner liegt mir 1 Exemplar aus 'Tumbatu vor, dats in den wesentlichen Charakteren mit $A$. thigrsoide's übereinstimmt, im (icsamthabitus aber ziemlich verschieden ist, indem die Stïmme mehrfich geteilt sind und die Polypen ziemlich gedrängt stehen. Ich habe es als

bezeichnet.

A. thyroides var. ramosa

Farbenangabe: Stamm haugrau, Köpfchen violettbraungrau.

\section{Ammothea baniana 11. sp.}

I) iagnose: Kolonie massig, derb. P'olypen in his $7 \mathrm{~mm}$ langen und $4 \mathrm{~mm}$ breiten dichten Kä̈zchen, $0,95 \mathrm{~mm}$ lang, $0,85 \mathrm{~mm}$ breit. Spicula sämtlich schlanke Spindeln mit wenigen Waren. Polypenspicula (),09 his $0,38 \mathrm{~mm}$ lang, (0,0)8-0,02) $\mathrm{mm}$ breit. Rindenspicula und Spicula der Kanalwände $0,38 \mathrm{~mm}$ lang, $0,03 \mathrm{~mm}$ breit.

Beschreibung: Ein massiger Strunk teilt sich oben in mehrere gedrungene İste, die die dichten bis $7 \mathrm{~mm}$ langen und 4 mm breiten eiförmigen Kätzchen tragen. Die Größe der L'olypen und der Nadeln ergiebt sich aus obenstehender Diagnose. Die Farbe der liolonie ist hiellgrau.

Fundort: Insel Baui; 1 Exemplar.

\section{Ammothea digitata n. sp.}

Diagnose: Mehrere aufrechte rigide Stämme an der Basis verschmolzen, teilen sich oben melsfiach in fingerförmige fortsitze. l'olypen ziemlich dicht auf den 4-20 mm langen fingerförmigen Fortsiitzen. warzenförmig. Polypenspicula $0,2 \mathrm{~mm}$ lang, $0,016 \mathrm{~mm}$ breit, gerade orler schwateh gehogene schlanke Spindeln. Rindenspicula 0,38 mm lang. (1.019 mm dick. meist bogenförnige schlanke spindeln. Spieula der Kanalwände chenso groß, aber meist gerade gestreckt.

Beschreibung: 3 aufrechte bis $9 \mathrm{~mm}$ hohe fleischige stamme sind mit ihren untern Teilen zu einem gs mm breiten Strunk verwathsen. Ilne Obertläche ist fein längsgerieft. Am obern Fnde teilen sie sich mehrfach in fingerfürmige Fortsätze von 4-20 $\mathrm{mm}$ Iä̈nge und 2-6 $\mathrm{mm}$ I)icke, die ziemlich dicht mit Polypen besetzt sind. Diese erscheinen als sehr 
niedrige kreisförmige Wälle. Die Farbe der Kolonie ist hellbraun. Die iibrigen Charaktere ergeben sich aus der Diagnose. Vielleicht ist diese Spezies mit Alcyonium flabellum Q. G. identisch.

Fundort: Sansibar, 4 Exemplare.

\section{Ammothea flava n. sp.}

Diagnose: Ein hoher aufrechter Stamm spaltet sich am obern Encle in mehrere Äste, die sich in die fingerförmigen $3-8 \mathrm{~mm}$ langen Kätzchen teilen. Polypen $0,76 \mathrm{~mm}$ lang und $0,57 \mathrm{~mm}$ breit. Polypenspicula $0,38 \mathrm{~mm}$ lange und $0,012 \mathrm{~mm}$ breite Spindeln. Rindenspicula meist halbkreisförmig gebogene Spindeln von $0,2 \mathrm{~mm}$ Sehnenlänge und $0,03 \mathrm{~mm}$ Dicke, mit langen stumpfen Dornen. Spicula der Kanalwände $0,475 \mathrm{~mm}$ lange, 0,024 mm breite Spindeln, gerade gestreckt.

Beschreibung: Der Stamm ist $83 \mathrm{~mm}$ hoch und bis $13 \mathrm{~mm}$ breit. Seine Oberfläche ist fein längsgerieft. Die P'olypen sind cylindrisch. Als F arbe der lebenden Kolonieen ist angegeben: Stamm ganz hellbraunfleischfarben, Köpfe etwas dunkler, Polypen oft fast weißlich. Die Farbe der Alkoholexemplare ist hellgelb. Im Utbrigen siehe Diagnose.

Fundort: Tumbatu, S. W. Riff, 3 Exemplare.

\section{Ammothea cervicornis n. sp.}

Diagnose: Ein schlanker Strunk teilt sich am obern Ende in mehrere Äste, an denen die Polypen locker angeordnet sitzen. Polypen bis $1,74 \mathrm{~mm}$ lang und $0,66 \mathrm{~mm}$ breit. Polypenspicula $0,19 \mathrm{~mm}$ lang, $0,01 \mathrm{~mm}$ breit. Rindenspicula und Spicula der Kanalwände schlanke Spindeln, 0,28 $\mathrm{mm}$ lang, 0,019 $\mathrm{mm}$ breit. Die F a rb e der Kolonie ist weiß.

Fundort: Sansibar, Bueni-Riff. Mehrere Exemplare.

\section{Ammothea tumbatuana n. sp.}

Diagnose: Am untern Ende verwachsene Stämme spalten sich am obern Ende in mehrere aufwärts strebende Äste, auf denen die l'olypen locker stehen. Polypen mit ausgestreckten Tentakeln $6 \mathrm{~mm}$ lang, 0,5 mm breit. Spicula fehlen.

Beschreibung: 2 am untern Ende verwachsene bis etwa $14 \mathrm{~mm}$ Höhe sterile Stämme spalten sich am obern Ende in mehrere aufwärts strebende bis $26 \mathrm{~mm}$ lange Äste, auf denen die Polypen locker stehen. Die Tentakeln sind etwa halb so lang als der ganze Polyp und mit einer einfachen Reihe von Fiedern versehen. Die Farbe der Kolonie im Leben ist graugrün, in Alkohol gelblichgrau. - Die Kolonie hat keine typische Ammotheenform, scheint aber den Ammotheen am nächsten zu stehen.

Fundort: Tumbatu. 


\section{Anmothea africana n. sp.}

I) iagnose: Molnere aufrechte, am untern linde verwachsene Stämme teilen sich am obern fincle in zahlileiche aufwärts strebonde $\tilde{A}$ ste, auf denen die $1 \mathrm{~mm}$ langen Polypen locker angeordnet sitzen. Rindenspicula und Spicula der Kanalwände fast glatte Spindeln, nur an beiden Fnden mit etwas stärkern Dornen verselen, $0.38 \mathrm{~mm}$ lang. Polypenspicula auf der ganzen Oberfläche mit deutlichen Warzen besetzt.

Beschreibung: Ier gröbte der Stämme ist 45 mu lioch und gegen $6 \mathrm{~mm}$ breit. Dic $\ddot{A}$ ste sind bis $10 \mathrm{~mm}$ lang und $2 \mathrm{~mm}$ dick. Iie Farbe der Kolonie ist hellgelb.

Fund ort: Tumbatu, S. Riff, sehr niedriges Wasser.

\section{Ammothea elegans n. sp.}

1) iagnose: Ier Stamm teilt sich am obern Finde in mehrere, sich wiederholt verzweigende Äste. P'olypen in schlanken Kätzchen, 1,16 mm lang, 0,85 $\mathrm{mm}$ breit. Polypenspicula 0,16 $\mathrm{mm}$ lang, $0,01 \mathrm{~mm}$ breit. Spicula der Kanalwände $0,38-0,47 \mathrm{~mm}$ lange, $0,03 \mathrm{~mm}$ breite, schlanke Spindeln, auf der ganzen Oberfläche mit feinen Warzen besetzt. Ebensolche in der Stammriude, außerdem sehr zahlreiche Doppelkugeln von $0,095 \mathrm{~mm}$ Längre und sehr kurzem $0,032 \mathrm{~mm}$ breiten Mittelstück.

Beschreibung: Der sterile Stamm ist $37 \mathrm{~mm}$ hoch und $25 \mathrm{~mm}$ breit und teilt sich am obern Ende in 4 Äste. Iie Kolonie ist im Leben grauviolett, in Alkohol grauweif.

Fundort: Tumbatu, Südriff. 1 Exemplar.

\section{Ammothea brassica n. sp.}

Diagnose: Kolonie blumenkohlartig. Polypen dichtgedrüngt am obern Ende der Äste, bis $2 \mathrm{~mm}$ lang und $1 \mathrm{~mm}$ breit. Polypenspicula 0,19-0,38 $\mathrm{mm}$ lang, $0,016 \mathrm{~mm}$ breit. Rindenspicula und Spicula der Kanalwände schlanke Spindeln mit ziemlich langen Warzen, $0,285-0,38 \mathrm{~mm}$ lang, 0,024 $\mathrm{mm}$ breit.

Beschreibung: Von gemeinsamer Basis erheben sich dichtgedrängt stehende einfache oder mehr oder weniger tief gespaltene $\ddot{A}$ ste ron durchschnittlich $30 \mathrm{~mm}$ Höhe. Die Längserstreckung der Kolonie beträgt $45 \mathrm{~mm}$. die Breite $30 \mathrm{~mm}$. Die Polypen stehen dichtgedrängt am obern Ende der Äste, mehr oder weniger tief herahgehend, die obersten sind rollstïndig in ihrer ganzen Länge mit einander rerwachsen. Die F a rbe der Kolonie ist grau. Im übrigen siehe Diagnose.

Fundort: Insel Baui. 1 Exemplar.

\section{Ammothea viridis n. sp.}

Diagnose: Ein massiger Strunk spaltet sich an obern Encle in mehrere vielfach reriistelte Zweige. Polypen in dichten Kiitzchen, $0.8 \mathrm{~mm}$ 
lang, 0,6 mm breit. Spicula fehlen in den Polypen und Ästen. Rindenspicula des untern Stammteils unregelmäßjig gestaltete Körper mit langen Dornen. Spicula der Kanalwände sehr plumpe Spinclehn, 0,5 mm lang, $0,1 \mathrm{~mm}$ dick.

Beschreibung: Die wesentlichen Charaktere ergeben sich aus der Diagnose. Der Strunk ist $45 \mathrm{~mm}$ hoch und ebenso breit. Die $/ / w$ wige sind bis $65 \mathrm{~mm}$ lang. Die Farbe der Kolonie ist grün.

Fundort: Insel Baui und Insel Muemba. 3 Exemplare.

\section{Ammothea stuhlmannii n. sp.}

Diagnose: '/ahllreiche platte sehr schlaffe Stämme auf gemeinsamer Membran, teilen sich oben wiederholt dichotomisch. Polypen in langgestreckten spitzen Kätzchen von $10-20 \mathrm{~mm}$ Länge und $2-4 \mathrm{~mm}$ Breite, $1,4 \mathrm{~mm}$ lang, $0,47 \mathrm{~mm}$ breit, ohne Spicula. Rindenspicula stabförmig oder unregelmäßig, mit sehr langen Dornen, 0,2 mm lang, 0,03 mm breit. Spicula der Kanalwände $0,7 \mathrm{~mm}$ lange, 0,09 $\mathrm{mm}$ dicke, dicht mit Warzen besetzte Spindeln.

Beschreibung: Die Stämme sind bis $180 \mathrm{~mm}$ hoch, an der Basis bis $30 \mathrm{~mm}$ breit. Die Farbe der Kolonie ist gellichweiß. Die übrigen Charaktere ergeben sich aus der Diagnose.

Fundort: Ostafrika. 2 große Exemplare.

Litteratur:

\section{Gattung: Spongodes Less.}

1) ESPER, Die Pflanzentiere. Nürnberg 1791-97. T. III, S. 49.

2) LAMARCK, Hist. nat. des anim. s. vert. Tome II, Paris 1816, S. 410.

3) LAMOUROUX, Exposition méthodique des genres de l'ordre des Polypiers, l'aris 1821.

4) BLAINVILLE, Manuel d'Actinologie, Paris 1834, S. 523.

5) EHRENBERG, Die Korallentiere des roten Niecres, Berlin 1834, S. 60.

6) LESSON, Mlustrations de Zoologie, Paris 1834, Pl. XXI.

7) DANA, Zoophytes, Philadelphia 1846, S. 625.

8) MILNE-EDWARDS, Hist. nat. des Corall. Tome I, Paris 1857, S. 128.

9) DANA, Synopsis, New-Haven 1859, S. 126.

10) GRAY, Description of some new species of Spongodes and of a new allied genus (Morchellana) in the collection of the British Museum. P'roc. Zool. Soe. Lond. 1862.

11) VERRILL, List of the Polyps and Corals sent by the Aluseum of Comparative Zoology to other institutions in exchange. Bull. of the Mus. of ('omprar. Zool, Harvard College, Cambridge, Vol. I, 1863-69, S. 39.

12) KLUNZINGFi, Die Koralltiere des roten Meeres, Teil I, Berlin 18\%7, S. 34.

13) WRIGHT and STUDER, Report on the Aleyonaria coll. by H.M. S. Chall. Chall. Rep. Bd. XXXI, 1889, S. XXV u. 191.

14) HOLM, Beiträge zur Kenntnis der Alcyonidengattung Spongodes Less. Zool. Jahrb. Abt. f. Syst. Bd. VIII, 1895, S. 10.

15) KÜKEメTHAL, Alcyonaceen von Ternate. Zool. Anz. No. 488 u. 489, 1895.

16) KÜKENTHAL, Alicyonaceen v. Ternate. Frkft. a. M. 1896. 
Synonyma: Alcyonium (pars) Esp. (1).

Xenia (pars) Lu. (2).

Neptaea Blatnv. (4).

Nephthya (pars) ErrbG. (5).

Spongodes LEss: (6).

Spoggodia DANA (7 u. 9).

Spoggodes M.-E. (8).

Spoggodes + Spoggodia + Morchellana Gr. (10).

Spongodes excl. Neplithya HoLm (14).

Diagnose: Polypen mit Stiitzbündel, in Bündeln oder einzeln.

Systematik: Kükenthas (16) unterscheidet folgende Gruppen innerhalb der Gattung Spongodes:

I. Subgenus: Sprongodin: Polvpen vereinzelt, nicht in Bündeln vereint, an langen cylindrischen Ästen.

II. Sulggenus: Sponyodes: Polypen in Bündeln rereint:

A. Glomeratae: P'olypenhündel auseinander geclrängt, elonso wie die Äste.

B. Umbellatac: Polypen in Dolden an den Spitzen der äussersten Zweige.

('. Divaricatae: Äste wie Polypenhündel anseinandergespreitzt.

1. Cylindratae: mit cylindrischen Ästen.

2. Foliatae: untere Äste blattförmig verbreitert.

Man kennt bis jetzt 69 spongodes-Arten und zwar:

Spongodia: 5

Spongodes 64

Glomeratae 22

Umbellatae 15

Divaricatae 27

Cylindratae 11

Foliatae 16.

Eine Ühersicht sümtlicher his zum Jahr 1896 beschriebenen Arten findet sich bei KOrExthat (16). Ine ('hataktere der von mir heschriehenen neuen Arten des Berliner Muserums habe ich in meiner größßern Aleyonaceenarbeit übersichtlich zusammengestellt.

\section{Divaricatae.}

\section{Cylindratae.}

\section{Spongodes mirabilis n. sp.}

Diagnose: P'olypen einzeln oder in kleinen Bündeln, 1,16 mm lang. $0.8 \mathrm{~mm}$ breit. Polvpenkëpfehen gegen den Stiel so weit umgebogen, dat.3 sich beide berühren. Polypenspicula unregehmälisig angeordnet, von ah- 
weichender Gestalt. Die klcinern sind langgestreckte Hanteln, 0,07 mm lang, 0,01 mm dick in der Mitte, 0,02 mm dick an den Enden: die größeren sind Stäbe mit langen stumpfen Dornen, bis $0,3 \mathrm{~mm}$ lang, 0,028 mm dick. Stützbündelspicula $2 \mathrm{~mm}$ lang, 0,15 $\mathrm{mm}$ dick, eins etwa $0,3 \mathrm{~mm}$ vorragend. Stammspicula 0,15-1,8 $\mathrm{mm}$ lang, 0,038-0,19 $\mathrm{mm}$ dick. Spicula der Kanalwände ebenso.

Beschreibung: Ein $17 \mathrm{~mm}$ hoher, $6 \mathrm{~mm}$ breiter Strunk teilt sich am obern Ende in 5 cylindrische Äste, deren längster $35 \mathrm{~mm}$ lang ist. Die Farbe der Kolonie ist grau. Im übrigen siehe Diagnose.

Fundort: Sansibar. 1 Exemplar. 


\section{Verzeichnis \\ der beschriebenen Arten.}

Clavulariidae.

Clavularia longissima n. sp. Kokotoni.

Clavularia gracitis n. sp. Tumbatu.

Clazularia flava n. sp. Sansibar.

Clavularia celebensis Hickson. Tumbatu.

Sympodium coerulerm EHrBg. Tumbatu.

Sympodium fulvum (Forsk.). Tumbatu.

Sympodium punctatum n. sp. Tumbatu.

Telestidae.

Coelogorgia palmosa (VAL): Sansibar, Tumbatu.

Tubiporidae.

Tubipora muleola Q. G. var. sansibarica nov. Sansibar.

Tubipora chamissonis Ehrbg. Sansibar.

Xeniidae.

Xenia umbellata Sムv. Tumbatu, Insel Baui.

Xenia tumbatuana n. sp. Tumbatu.

Xenia elisabethae (KöLL). Sansibar.

Xenia fuscescens EHRBg. Sansibar.

Xenia membranacea ScHenk. Sansibar.

Xenia quinqueserta n. sp. Tumbatu.

Xenia baviana n. sp. Insel Baui.

Xenia medusoides n. sp. Tumbatu.

Cespitularia coeruleu n. sp. Kokotoni.

Alcyoniidae.

Sinularia brassica n. sp. Tumbatu.

Alcyonium polydactylum (Кнгвs.). Sansil)ar, Insel Baui.

Sarcophytum glaucum (Q. G.). Kokotoni, Tumbatu, Insel ('hangu.

Surcopliytum trocheliophonum Marexz, var. amboinense Marliaxz. Kokototoni. Sansibar.

Lobophyhum crassum Marexz, rar, sansibaricum nov. Sausibar, Tumbitu. 


$$
\text { Nephthyidae. }
$$

Ammothea thyrsoides EHRBG. Tumbatu.

Ammothea thyrsoides, var. ramosa nov. Tumbatu, leg. Stuhlmann.

Ammothea bariana n. sp. Insel Baui.

Ammothea digitata n. sp. Sansibar.

Ammothea flava n. sp. Tumbatu.

Ammothea cervicornis n. sp. Sansibar, Bueni Riff.

Ammothea tumbatuana n. sp. Tumbatu.

Ammothea africana $\mathrm{n}$. sp. Tumbatu.

Ammothea elegans n. sp. Tumbatu.

Ammothea brassica n. sp. Insel Baui.

Ammothea viridis n. sp. Insel Baui, Insel Muemba.

Ammothea stuhlmannii $\mathrm{n}$. sp. Ostafrika.

Spongodes mirabilis n. sp. Sansibar. 


\section{Lebensabriss.}

Walther May wurde am 12. Juni 1868 zu Marburg als Sohn des Hauptmanns ALEXIS MaY geboren, besuchte das Realgymnasium zu Cassel, rerlief3 es mit dem Zeugniß3 der Reife und studierte Naturwissenschaft in Jena. Seine Lehrer waren: v. Bardeleben, Detmer, JUrbringer, Haeckel, KÓkenthal, Linck, Stahl, Verworn und Walther, denen er hiermit seinen wärmsten Dank sagt. 





\section{PLEASE DO NOT REMOVE} CARDS OR SLIPS FROM THIS POCKET

\section{UNIVERSITY OF TORONTO LIBRARY}

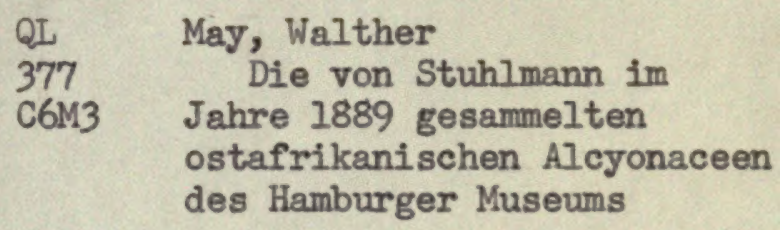

BioMed 
